\title{
Duality Bounds on the Cut-Off Rate with Applications to Ricean Fading
}

\author{
Amos Lapidoth Natalia Miliou
}

\begin{abstract}
We propose a technique to derive upper bounds on Gallager's cost-constrained random coding exponent function. Applying this technique to the non-coherent peak-power or average-power limited discrete time memoryless Ricean fading channel, we obtain the high signal-to-noise ratio (SNR) expansion of this channel's cut-off rate. At high SNR the gap between channel capacity and the cut-off rate approaches a finite limit. This limit is approximately 0.26 nats per channel-use for zero specular component (Rayleigh) fading and approaches 0.39 nats per channel-use for very large specular components.

We also compute the asymptotic cut-off rate of a Rayleigh fading channel when the receiver has access to some partial side information concerning the fading. It is demonstrated that the cut-off rate does not utilize the side information as efficiently as capacity, and that the high SNR gap between the two increases to infinity as the imperfect side information becomes more and more precise.
\end{abstract}

KEYwORDS: Asymptotic, channel capacity, cut-off rate, fading, high SNR, Ricean fading.

\section{Introduction}

This paper addresses the computation of a function that is key to the evaluation of both the random coding and sphere packing error exponents. This function, often denoted $E_{0}(\varrho)$, is usually expressed as a maximization problem over input distributions. Consequently, it is conceptually easily bounded from below: any feasible input distribution gives rise to such a bound. In this paper we propose to use a dual expression for $E_{0}(\varrho)$ - an expression that involves a minimization over output distributions - in order to derive upper bounds on $E_{0}(\varrho)$. We shall demonstrate this approach by studying 
the cutoff rate of non-coherent Ricean fading channels. To that end we shall have to study the appropriate modifications to the function $E_{0}(\varrho)$ that are needed to account for input constraints and when the channel input and output alphabets are infinite.

It should be noted that the dual expression we propose to use is not new 1], 2, Ex. 23 in Ch. 2.5]. We merely extend it here to input constrained channels over infinite alphabets and demonstrate how it can be used to derive analytic upper bounds on the random coding and sphere packing error exponents. For numerical procedures (for unconstrained finite alphabet channels) see [3].

The rest of this introductory section is dedicated to the introduction of the function $E_{0}(\varrho)$ for discrete memoryless channels. We first treat unconstrained channel and then introduce the modifications that are needed to account for input constraints. We describe both the "method of types" approach and Gallager's approach. We pay special attention to the modification that Gallager introduced to account for cost constraints and to the duality between the expressions derived using the two approaches. This introduction is somewhat lengthy because, while the results are not new, we had difficulty pointing to a publication that introduces the two approaches side by side and that compares the two in the presence of cost constraints.

In Section 2 we extend the discussion to infinite alphabets and prove the basic inequality on which our approach to upper bounding $E_{0}(\varrho)$ is based; see Proposition [1. In Section 3 we introduce the discrete-time memoryless Ricean fading channel with and without full or partial side information at the receiver, and we describe our asymptotic results on this channel's cutoff rate. These asymptotic results are derived using duality in Section 4, which concludes the paper.

\subsection{Unconstrained Inputs}

To motivate the interest in the function $E_{0}(\varrho)$ we shall begin by addressing the case where there are no input constraints. The reliability function $E(R)$ corresponding to rate- $R$ unconstrained communication over a discrete memoryless channel (DMC) of capacity $C \geq R$ is the best exponential decay in the blocklength $n$ of the average probability of error that one can achieve using rate- $R$ blocklength- $n$ codebooks. That is,

$$
E(R) \triangleq \varlimsup_{n \rightarrow \infty}-\frac{1}{n} \log \mathrm{P}_{\mathrm{e}}(n, R)
$$

where $\mathrm{P}_{\mathrm{e}}(n, R)$ denotes the average probability of error of the best rate- $R$ blocklength- $n$ codebook for the given channel. 
The problem of computing the reliability function of a general DMC over the finite input and output alphabets $\mathcal{X}$ and $\mathcal{Y}$ and of a general law $\mathrm{W}(y \mid x)$ is still open. Various upper and lower bounds are, however, known. To derive lower bounds on the reliability function one must derive upper bounds on the probability of error of the best rate- $R$ blocklength- $n$ code. This is typically done by demonstrating the existence of good codes for which the average probability of error is small. One such lower bound on $E(R)$ is the random coding lower bound [4]. By considering an ensemble of codebooks whose codewords are chosen independently, each according to a product distribution of marginal law Q, Gallager derived the lower bound

$$
E(R) \geq E_{\mathrm{G}}(R, \mathrm{Q})
$$

where

$$
E_{\mathrm{G}}(R, \mathrm{Q}) \triangleq \max _{0 \leq \varrho \leq 1}\left\{E_{\mathrm{G}, 0}(\varrho, \mathrm{Q})-\varrho R\right\}
$$

and

$$
E_{\mathrm{G}, 0}(\varrho, \mathrm{Q}) \triangleq-\log \sum_{y \in \mathcal{Y}}\left(\sum_{x \in \mathcal{X}} \mathrm{Q}(x) \mathrm{W}(y \mid x)^{\frac{1}{1+\varrho}}\right)^{1+\varrho} .
$$

Since the law $\mathrm{Q}$ from which the ensemble of codebooks is constructed is arbitrary, Gallager obtained the bound

$$
E(R) \geq E_{\mathrm{G}, \mathrm{r}}(R)
$$

where $E_{\mathrm{G}, \mathrm{r}}(R)$ is Gallager's random coding error exponent

$$
\begin{aligned}
E_{\mathrm{G}, \mathrm{r}}(R) & \triangleq \max _{\mathrm{Q}} E_{\mathrm{G}}(R, \mathrm{Q}) \\
& =\max _{\mathrm{Q}} \max _{0 \leq \varrho \leq 1}\left\{E_{\mathrm{G}, 0}(\varrho, \mathrm{Q})-\varrho R\right\} .
\end{aligned}
$$

A different random coding lower bound on the reliability function can be derived using the ensemble of codebooks where the codewords are still chosen independently, but rather than according to a product distribution, each is now chosen uniformly over a type class [2, 2.5], [1], [5]. With this approach one obtains [2, 2.5], [1] the lower bound

$$
E(R) \geq E_{\mathrm{CK}}(R, \mathrm{Q})
$$

where

$$
E_{\mathrm{CK}}(R, \mathrm{Q}) \triangleq \min _{\mathrm{V}(\cdot \mid \cdot)}\left\{D(\mathrm{~V} \| \mathrm{W} \mid \mathrm{Q})+|I(\mathrm{Q}, \mathrm{V})-R|^{+}\right\}
$$


Here the minimization is over all conditional laws

$$
\begin{aligned}
& \mathrm{V}(y \mid x) \geq 0, \quad \sum_{y \in \mathcal{Y}} \mathrm{V}(y \mid x)=1, \forall x \in \mathcal{X} \\
& D(\mathrm{~V} \| \mathrm{W} \mid \mathrm{Q})=\sum_{x \in \mathcal{X}} \mathrm{Q}(x) D(\mathrm{~V}(\cdot \mid x) \| \mathrm{W}(\cdot \mid x)) \\
&=\sum_{x \in \mathcal{X}} \mathrm{Q}(x) \sum_{y \in \mathcal{Y}} \mathrm{V}(y \mid x) \log \frac{\mathrm{V}(y \mid x)}{\mathrm{W}(y \mid x)}
\end{aligned}
$$

the term $I(\mathrm{Q}, \mathrm{V})$ denotes the mutual information corresponding to the channel $\mathrm{V}$ and the input distribution $\mathrm{Q}$; and $|\xi|^{+}$stands for $\max \{\xi, 0\}$. Again, since the type $Q$ according to which the ensemble is generated is arbitrary, one obtains

$$
E(R) \geq E_{\mathrm{CK}, \mathrm{r}}(R)
$$

where

$$
\begin{aligned}
E_{\mathrm{CK}, \mathrm{r}}(R) & \triangleq \max _{\mathrm{Q}} E_{\mathrm{CK}}(R, \mathrm{Q}) \\
& =\max _{\mathrm{Q}} \min _{\mathrm{V}(\cdot \mid \cdot)}\left\{D(\mathrm{~V} \| \mathrm{W} \mid \mathrm{Q})+|I(\mathrm{Q}, \mathrm{V})-R|^{+}\right\} .
\end{aligned}
$$

There is an alternative form for $E_{\mathrm{CK}}(R, \mathrm{Q})$ that will be of interest to us [1], [2, Ex. 23 in Ch. 2.5]. This form is more similar to (3):

$$
E_{\mathrm{CK}}(R, \mathrm{Q})=\max _{0 \leq \varrho \leq 1}\left\{E_{\mathrm{CK}, 0}(\varrho, \mathrm{Q})-\varrho R\right\}
$$

where

$$
\begin{aligned}
E_{\mathrm{CK}, 0}(\varrho, \mathrm{Q}) & \triangleq \min _{\mathrm{V}(\cdot \mid \cdot)}\{D(\mathrm{~V} \| \mathrm{W} \mid \mathrm{Q})+\varrho I(\mathrm{Q}, \mathrm{V})\} \\
& =\min _{\mathrm{R}}\left\{-(1+\varrho) \sum_{x \in \mathcal{X}} \mathrm{Q}(x) \log \left(\sum_{y \in \mathcal{Y}} \mathrm{W}(y \mid x)^{\frac{1}{1+\varrho}} \mathrm{R}(y)^{\frac{\varrho}{1+\varrho}}\right)\right\}
\end{aligned}
$$

and where the minimization in the latter is over the set of all distributions $\mathrm{R}$ on the output alphabet $\mathcal{Y}$.

In general, for any DMC W $(y \mid x)$ and any input distribution Q [1], 2, Ex. 23 in Ch. 2.5]

$$
E_{\mathrm{CK}, 0}(\varrho, \mathrm{Q}) \geq E_{\mathrm{G}, 0}(\varrho, \mathrm{Q}), \quad \varrho \geq 0
$$

and hence

$$
E_{\mathrm{CK}}(R, \mathrm{Q}) \geq E_{\mathrm{G}}(R, \mathrm{Q})
$$


with the inequalities typically being strict. These inequalities are a consequence of the fact that the "average constant composition code" performs better than the "average independent and identically distributed code" [6] . However, when optimized over the input distributions, the inequalities turn into equalities [1], 7], 2, Ex. 23 in Ch. 2.5]

$$
\max _{\mathrm{Q}} E_{\mathrm{CK}, 0}(\varrho, \mathrm{Q})=\max _{\mathrm{Q}} E_{\mathrm{G}, 0}(\varrho, \mathrm{Q}), \quad \varrho \geq 0
$$

and

$$
\max _{\mathbf{Q}} E_{\mathrm{CK}}(R, \mathrm{Q})=\max _{\mathbf{Q}} E_{\mathrm{G}}(R, \mathrm{Q})
$$

i.e.,

$$
E_{\mathrm{CK}, \mathrm{r}}(R)=E_{\mathrm{G}, \mathrm{r}}(R) .
$$

In fact, as shown in Appendix $\mathrm{A}$, the optimization problems appearing on the LHS and on the RHS of (22) are Lagrange duals.

Consequently, we shall henceforth denote $\max _{\mathrm{Q}} E_{\mathrm{CK}, 0}(\varrho, \mathrm{Q})\left(=\max _{\mathrm{Q}} E_{\mathrm{G}, 0}(\varrho, \mathrm{Q})\right)$ by $E_{0}(\varrho)$ and refer to $E_{\mathrm{G}, \mathrm{r}}(R)\left(=E_{\mathrm{CK}, \mathrm{r}}(R)\right)$ as the random coding error exponent and denote it by $E_{\mathrm{r}}(R)$. In terms of the function $E_{0}(\cdot)$ the random coding error exponent $E_{\mathrm{r}}(R)$ is thus given by

$$
E_{\mathrm{r}}(R)=\max _{0 \leq \varrho \leq 1}\left\{E_{0}(\varrho)-\varrho R\right\} .
$$

The cut-off rate $R_{0}$ is defined by

$$
R_{0}=\left.E_{0}(\varrho)\right|_{\varrho=1} .
$$

The function $E_{0}(\varrho)$ also plays an important role in the study of upper bounds to the reliability function. In fact, the sphere packing error exponent $E_{\mathrm{sp}}(R)$ is given by [4]

$$
E_{\mathrm{sp}}(R)=\max _{\varrho \geq 0}\left\{E_{0}(\varrho)-\varrho R\right\} .
$$

Combining (21) with (18) and (41) we obtain the two equivalent expressions for $E_{0}(\varrho)$

$$
\begin{gathered}
E_{0}(\varrho)=\max _{\mathrm{Q}}\left\{-\log \sum_{y \in \mathcal{Y}}\left(\sum_{x \in \mathcal{X}} \mathrm{Q}(x) \mathrm{W}(y \mid x)^{\frac{1}{1+\varrho}}\right)^{1+\varrho}\right\} \\
E_{0}(\varrho)=\max _{\mathrm{Q}} \min _{\mathrm{R}}\left\{-(1+\varrho) \sum_{x \in \mathcal{X}} \mathrm{Q}(x) \log \left(\sum_{y \in \mathcal{Y}} \mathrm{W}(y \mid x)^{\frac{1}{1+\varrho}} \mathrm{R}(y)^{\frac{\varrho}{1+\varrho}}\right)\right\} .
\end{gathered}
$$


We refer to the former expression as the "primal" expression and to the latter as the "dual" expression. The primal expression is useful for the derivation of lower bounds on $E_{0}(\varrho)$. Indeed, any distribution $Q$ on the input alphabet $\mathcal{X}$ induces the lower bound

$$
E_{0}(\varrho) \geq-\log \sum_{y \in \mathcal{Y}}\left(\sum_{x \in \mathcal{X}} \mathrm{Q}(x) \mathrm{W}(y \mid x)^{\frac{1}{1+\varrho}}\right)^{1+\varrho} .
$$

On the other hand, the dual expression is useful for the derivation of upper bounds. Any distribution $\mathrm{R}$ on the output alphabet $\mathcal{Y}$ yields the upper bound

$$
\begin{aligned}
E_{0}(\varrho) & \leq \max _{\mathrm{Q}}\left\{-(1+\varrho) \sum_{x \in \mathcal{X}} \mathrm{Q}(x) \log \left(\sum_{y \in \mathcal{Y}} \mathrm{W}(y \mid x)^{\frac{1}{1+\varrho}} \mathrm{R}(y)^{\frac{\varrho}{1+\varrho}}\right)\right\} \\
& =\max _{x \in \mathcal{X}}\left\{-(1+\varrho) \log \left(\sum_{y \in \mathcal{Y}} \mathrm{W}(y \mid x)^{\frac{1}{1+\varrho}} \mathrm{R}(y)^{\frac{\varrho}{1+\varrho}}\right)\right\} .
\end{aligned}
$$

\subsection{Constrained Inputs}

Before we can use the above bounds for fading channels we need to extend the discussion to cost constrained channels and to channels over infinite input and output alphabets where the method of types cannot be directly used. For now we continue our assumption of finite alphabets and address the cost constraint.

Suppose we limit ourselves to blockcode transmissions where we only allow codewords $\left(x_{1}, \ldots, x_{n}\right)$ that satisfy

$$
\sum_{\ell=1}^{n} g\left(x_{\ell}\right) \leq n \Upsilon
$$

where $g: \mathcal{X} \rightarrow \mathbb{R}^{+}$is a cost function on the input alphabet $\mathcal{X}, \Upsilon$ is some prespecified non-negative number, and $n$, as before, is the blocklength. The reliability function $E(R)$ is defined as in (11) with the modification that $\mathrm{P}_{\mathrm{e}}(n, R)$ should be now understood as the lowest average probability of error that can be achieved using a rate- $R$ blocklength- $n$ codebook all of whose codewords satisfy the cost constraint.

To obtain lower bounds on $E(R)$ Gallager [4, 8] modified his random coding argument in two ways. He introduced a new ensemble of codebooks and introduced an improved technique to analyze the average probability of error over this ensemble. For any probability law $Q$ on the input alphabet satisfying

$$
\mathrm{E}_{\mathrm{Q}}[g(X)] \leq \Upsilon
$$


where

$$
\mathrm{E}_{\mathrm{Q}}[g(X)] \triangleq \sum_{x \in \mathcal{X}} \mathrm{Q}(x) g(x)
$$

define

$$
E_{\mathrm{G}, 0}^{\mathrm{M}}(\varrho, \mathrm{Q}) \triangleq \begin{cases}E_{\mathrm{G}, 0}(\varrho, \mathrm{Q}) & \text { if } \mathrm{E}_{\mathrm{Q}}[g(X)]<\Upsilon \\ \max _{r \geq 0} E_{0}(\varrho, \mathrm{Q}, r) & \text { if } \mathrm{E}_{\mathrm{Q}}[g(X)]=\Upsilon\end{cases}
$$

where

$$
E_{0}(\varrho, \mathrm{Q}, r) \triangleq-\log \sum_{y \in \mathcal{Y}}\left(\sum_{x \in \mathcal{X}} \mathrm{Q}(x) e^{r(g(x)-\Upsilon)} \mathrm{W}(y \mid x)^{\frac{1}{1+\varrho}}\right)^{1+\varrho}
$$

Note that

$$
\left.E_{0}(\varrho, \mathrm{Q}, r)\right|_{r=0}=E_{\mathrm{G}, 0}(\varrho, \mathrm{Q})
$$

and hence

$$
\max _{r \geq 0} E_{0}(\varrho, \mathrm{Q}, r) \geq E_{\mathrm{G}, 0}^{\mathrm{M}}(\varrho, \mathrm{Q}) \geq E_{\mathrm{G}, 0}(\varrho, \mathrm{Q}) .
$$

Thus, Gallager's "modification" can only tighten the bound.

Gallager then showed that for any $0 \leq \varrho \leq 1$ the exponent

$$
E_{\mathrm{G}, 0}^{\mathrm{M}}(\varrho, \mathrm{Q})-\varrho R
$$

is achievable using block codes that satisfy the constraint.

(To prove this result when $\mathrm{E}_{\mathrm{Q}}[g(X)]<\Upsilon$ he considered an ensemble of codebooks where the codewords are chosen independently of each other, each according to the a-posteriori law of a sequence $X_{1}, \ldots, X_{n}$ drawn IID according to $\mathrm{Q}$ conditional on $\sum_{k=1}^{n} g\left(X_{k}\right) \leq n \Upsilon$. To prove the result when $\mathrm{E}_{\mathrm{Q}}[g(X)]=\Upsilon$ he considered an ensemble similarly constructed but with the distribution being conditional on $n \Upsilon-\delta \leq \sum_{k=1}^{n} g\left(X_{k}\right) \leq n \Upsilon$.)

Consequently the error exponent

$$
E_{\mathrm{G}, \mathrm{r}}^{\mathrm{M}}(R, \Upsilon) \triangleq \max _{0 \leq \varrho \leq 1}\left\{E_{\mathrm{G}, 0}^{\mathrm{M}}(\varrho, \Upsilon)-\varrho R\right\}
$$

where

$$
E_{\mathrm{G}, 0}^{\mathrm{M}}(\varrho, \Upsilon) \triangleq \max _{\mathrm{Q}: \mathrm{E}_{\mathrm{Q}}[g(X)] \leq \Upsilon} E_{\mathrm{G}, 0}^{\mathrm{M}}(\varrho, \mathrm{Q})
$$

is achievable.

It is instructive to distinguish between two types of constraints. We say that the cost constraint is inactive if there exists some input distribution $\mathrm{Q}^{*}$ 
satisfying the constraint that achieves the global unconstrained maximum of $E_{\mathrm{G}, 0}(\varrho, \mathrm{Q})$. That is,

$$
\exists \mathrm{Q}^{*}: \mathrm{E}_{\mathrm{Q}^{*}}[g(X)] \leq \Upsilon \quad \text { and } \quad E_{\mathrm{G}, 0}\left(\varrho, \mathrm{Q}^{*}\right)=\max _{\mathrm{Q}} E_{\mathrm{G}, 0}(\varrho, \mathrm{Q})
$$

or equivalently

$$
\max _{\mathrm{Q}: \mathrm{E}_{\mathrm{Q}}[g(X)] \leq \Upsilon} E_{\mathrm{G}, 0}(\varrho, \mathrm{Q})=\max _{\mathrm{Q}} E_{\mathrm{G}, 0}(\varrho, \mathrm{Q}) .
$$

Otherwise, we say that the cost constraint is active. With these definitions it can be shown that (40) simplifies to

$$
E_{\mathrm{G}, 0}^{\mathrm{M}}(\varrho, \Upsilon)=\left\{\begin{array}{ll}
\max _{\mathrm{Q}: \mathrm{E}_{\mathrm{Q}}[g(X)]=\Upsilon} \max _{r \geq 0} E_{0}(\varrho, \mathrm{Q}, r) & \text { cost active } \\
\max _{\mathrm{Q}} E_{\mathrm{G}, 0}(\varrho, \mathrm{Q}) & \text { cost inactive }
\end{array} .\right.
$$

(The case where the cost constraint is active follows from Gallager's observation that when the cost constraint is active, the maximum of $E_{0}(\varrho, Q, r)$ over all $r \geq 0$ and over all laws $Q$ satisfying (33) is achieved by an input distribution $Q_{*}$ satisfying the constraint with equality. The case where the cost constraint is inactive follows by noting that by starting from (38) we have for inactive cost constraints

$$
\begin{aligned}
\max _{\mathrm{Q}: \mathrm{E}_{\mathrm{Q}}[g(X)] \leq \Upsilon} E_{\mathrm{G}, 0}^{\mathrm{M}}(\varrho, \mathrm{Q}) & \geq \max _{\mathrm{Q}: \mathrm{E}_{\mathrm{Q}}[g(X)] \leq \Upsilon} E_{\mathrm{G}, 0}(\varrho, \mathrm{Q}) \\
& =\max _{\mathrm{Q}} E_{\mathrm{G}, 0}(\varrho, \mathrm{Q}) \\
& =\max _{\mathrm{Q}} E_{\mathrm{CK}, 0}(\varrho, \mathrm{Q}) \\
& \geq \max _{\mathrm{Q}: \mathrm{E}_{\mathrm{Q}}[g(X)] \leq \Upsilon} E_{\mathrm{G}, 0}^{\mathrm{M}}(\varrho, \mathrm{Q})
\end{aligned}
$$

so that all inequalities must hold with equalities. Here the first inequality follows from (38); the subsequent equality because the cost constraint is assumed inactive (42); the subsequent equality from (21); and the final inequality from (46) ahead.)

An achievable error exponent can also be demonstrated using constant composition codes. This yields that the error exponent

$$
E_{\mathrm{CK}, \mathrm{r}}(R, \Upsilon) \triangleq \max _{0 \leq \varrho \leq 1}\left\{E_{\mathrm{CK}, 0}(\varrho, \Upsilon)-\varrho R\right\}
$$

is achievable where

$$
E_{\mathrm{CK}, 0}(\varrho, \Upsilon) \triangleq \max _{\mathrm{Q}: \mathrm{E}_{\mathrm{Q}}[g(X)] \leq \Upsilon} E_{\mathrm{CK}, 0}(\varrho, \mathrm{Q})
$$


The relation (38) not withstanding, it can be shown that for any law $Q$ satisfying (33) and any $\varrho \geq 0$

$$
E_{\mathrm{CK}, 0}(\varrho, \mathrm{Q}) \geq E_{\mathrm{G}, 0}^{\mathrm{M}}(\varrho, \mathrm{Q})
$$

with the inequality being, in general, strict. ${ }^{1}$ Consequently, by (45) and (40) we have $E_{\mathrm{CK}, 0}(\varrho, \Upsilon) \geq E_{\mathrm{G}, 0}^{\mathrm{M}}(\varrho, \Upsilon)$. However, as shown in Appendix B this holds with equality

$$
E_{\mathrm{CK}, 0}(\varrho, \Upsilon)=E_{\mathrm{G}, 0}^{\mathrm{M}}(\varrho, \Upsilon)
$$

Thus, denoting the two identical functions $E_{\mathrm{G}, 0}^{\mathrm{M}}(\varrho, \Upsilon)$ and $E_{\mathrm{CK}, 0}(\varrho, \Upsilon)$ by $E_{0}(\varrho, \Upsilon)$ and the two identical functions $E_{\mathrm{CK}, \mathrm{r}}(R, \Upsilon)$ and $E_{\mathrm{G}, \mathrm{r}}^{\mathrm{M}}(R, \Upsilon)$ by $E_{\mathrm{r}}(R, \Upsilon)$ we have

$$
E_{\mathrm{r}}(R, \Upsilon)=\max _{0 \leq \varrho \leq 1}\left\{E_{0}(\varrho, \Upsilon)-\varrho R\right\}
$$

where $E_{0}(\varrho, \Upsilon)$ can be expressed either by (43) as

$$
E_{0}(\varrho, \Upsilon)= \begin{cases}\max _{\mathrm{Q}: E_{\mathrm{Q}}[g(X)]=\Upsilon} \max _{r \geq 0} E_{0}(\varrho, \mathrm{Q}, r) & \text { cost active } \\ \max _{\mathrm{Q}} E_{\mathrm{G}, 0}(\varrho, \mathrm{Q}) & \text { cost inactive }\end{cases}
$$

or, using (18), as

$$
\begin{aligned}
& E_{0}(\varrho, \Upsilon)= \\
& \max _{\mathrm{Q}: \mathrm{E}_{\mathrm{Q}}[g(X)] \leq \Upsilon} \min _{\mathrm{R}}\left\{-(1+\varrho) \sum_{x \in \mathcal{X}} \mathrm{Q}(x) \log \left(\sum_{y \in \mathcal{Y}} \mathrm{W}(y \mid x)^{\frac{1}{1+\varrho}} \mathrm{R}(y)^{\frac{\varrho}{1+\varrho}}\right)\right\} .
\end{aligned}
$$

The former, to which we refer as the "primal" expression, is useful for the derivation of lower bounds on $E_{0}(\varrho, \Upsilon)$ whereas the latter, the "dual", is useful for upper bounds.

\section{Continuous Alphabets}

We next extend the discussion to channels over infinite input and output alphabets. Consider a channel $W(\cdot \mid \cdot)$ whose inputs and outputs take value in the separable metric spaces $\mathcal{X}$ and $\mathcal{Y}$ respectively. Thus for any input $x \in \mathcal{X}$ and any Borel set $\mathcal{B} \subset \mathcal{Y}$ the probability that in response to the input $x$ the channel will produce an output $Y$ that lies in the set $\mathcal{B}$ is $W(\mathcal{B} \mid x)$. We

\footnotetext{
${ }^{1}$ In the case $\mathrm{E}_{\mathrm{Q}}[g(X)]<\Upsilon$ this follows directly from (20). For a proof in the case $\mathrm{E}_{\mathbf{Q}}[g(X)]=\Upsilon$ see Proposition 1 ahead, which proves that the RHS of (18) is greater or equal $E_{\mathrm{G}, 0}^{\mathrm{M}}(\varrho, \mathrm{Q})$.
} 
assume that the mapping $x \mapsto W(\mathcal{B} \mid x)$ from $\mathcal{X}$ to the interval $[0,1]$ is Borel measurable. Finally assume the existence of an underlying positive measure $\mu$ on $\mathcal{Y}$ with respect to which all the probability measures $\{W(\cdot \mid x), x \in \mathcal{X}\}$ are absolutely continuous. Denote the Radon-Nykodim derivative of $W(\cdot \mid x)$ with respect to $\mu$ by

$$
w(\cdot \mid x)=\frac{\mathrm{d} W(\cdot \mid x)}{\mathrm{d} \mu}, \quad x \in \mathcal{X}
$$

Thus, $w(y \mid x)$ is the density at $y$ of the channel output corresponding to the input $x \in \mathcal{X}$. For any input $x \in \mathcal{X}$ and any Borel set $\mathcal{B} \subset \mathcal{Y}$

$$
W(\mathcal{B} \mid x)=\int_{\mathcal{B}} w(y \mid x) \mathrm{d} \mu(y) .
$$

As to the cost, we shall assume that the function $g: \mathcal{X} \rightarrow \mathbb{R}^{+}$is measurable and consider block codes that satisfy (32). We extend the definition (34) to infinite alphabets as

$$
\mathrm{E}_{\mathbf{Q}}[g(X)] \triangleq \int_{\mathcal{X}} g(x) \mathrm{dQ}(x)
$$

Definition (36) is extended for any probability law $\mathrm{Q}$ on $\mathcal{X}$ as

$$
E_{0}(\varrho, \mathrm{Q}, r) \triangleq-\log \int_{y \in \mathcal{Y}}\left(\int_{x \in \mathcal{X}} e^{r(g(x)-\Upsilon)} w(y \mid x)^{\frac{1}{1+\varrho}} \mathrm{dQ}(x)\right)^{1+\varrho} \mathrm{d} \mu(y) .
$$

For any input distribution $\mathrm{Q}$ satisfying the constraint $\mathrm{E}_{\mathrm{Q}}[g(X)] \leq \Upsilon$ we extend (35) as follows:

$$
E_{\mathrm{G}, 0}^{\mathrm{M}}(\varrho, \mathrm{Q}) \triangleq \begin{cases}\sup _{r \geq 0} E_{0}(\varrho, \mathrm{Q}, r) & \text { if } \mathrm{E}_{\mathrm{Q}}[g(X)]=\Upsilon \text { and } \mathrm{E}_{\mathrm{Q}}\left[g^{3}(X)\right]<\infty \\ \left.E_{0}(\varrho, \mathrm{Q}, r)\right|_{r=0} & \text { otherwise }\end{cases}
$$

(Note that following Gallager [4], [8] we allow for the optimization over $r$ only when under the law $\mathrm{Q}$ the random variable $g(X)$ has a finite third moment.)

With this definition we can now define

$$
E_{0}(\varrho, \Upsilon) \triangleq \sup _{\mathrm{Q}: \mathrm{E}_{\mathrm{Q}}[g(X)] \leq \Upsilon} E_{\mathrm{G}, 0}^{\mathrm{M}}(\varrho, \mathrm{Q})
$$

and the cut-off rate as

$$
\left.R_{0}(\Upsilon) \triangleq E_{0}(\varrho, \Upsilon)\right|_{\varrho=1}
$$


The random coding error exponent

$$
\sup _{0 \leq \varrho \leq 1}\left\{E_{0}(\varrho, \Upsilon)-\varrho R\right\}
$$

is achievable with block codes satisfying the constraint (32) [4, 8].

The following proposition proves (46) in the more general case where the alphabets may be continuous. It is particularly useful for the derivation of upper bounds on $E_{\mathrm{G}, 0}^{\mathrm{M}}(\varrho, \Upsilon)$.

Proposition 1. Consider as above a discrete-time memoryless infinite alphabet channel $w(y \mid x)$, an output measure $\mu$, a measurable cost function $g: \mathcal{X} \rightarrow \mathbb{R}^{+}$, and some arbitrary allowed cost $\Upsilon$. Let $f_{R}$ be an arbitrary density with respect to $\mu$ on the output alphabet $\mathcal{Y}$. Then for any distribution $\mathrm{Q}$ on $\mathcal{X}$ satisfying the cost constraint $\mathrm{E}_{\mathrm{Q}}[g(X)] \leq \Upsilon$

$$
\begin{aligned}
E_{\mathrm{G}, 0}^{\mathrm{M}}(\varrho, \mathrm{Q}) & \leq \\
& -(1+\varrho) \int_{x \in \mathcal{X}} \log \left(\int_{y \in \mathcal{Y}} w(y \mid x)^{\frac{1}{1+\varrho}} f_{R}(y)^{\frac{\varrho}{1+\varrho}} \mathrm{d} \mu(y)\right) \mathrm{dQ}(x) .
\end{aligned}
$$

Proof. Distinguish between the case where $\mathrm{E}_{\mathrm{Q}}[g(X)]<\Upsilon$ and the case where $\mathrm{E}_{\mathrm{Q}}[g(X)]=\Upsilon$ and $\mathrm{E}_{\mathrm{Q}}\left[g^{3}(X)\right]<\infty$. In the former case, by (54), $E_{\mathrm{G}, 0}^{\mathrm{M}}(\varrho, \mathrm{Q})=$ $E_{0}(\varrho, Q, 0)$ and the result follows by an application of Jensen's inequality and Hölder's inequality:

$$
\begin{aligned}
& -(1+\varrho) \int_{x \in \mathcal{X}} \log \left(\int_{y \in \mathcal{Y}} w(y \mid x)^{\frac{1}{1+\varrho}} f_{R}(y)^{\frac{\varrho}{1+\varrho}} \mathrm{d} \mu(y)\right) \mathrm{dQ}(x) \\
& \geq-(1+\varrho) \log \int_{x \in \mathcal{X}} \int_{y \in \mathcal{Y}} w(y \mid x)^{\frac{1}{1+\varrho}} f_{R}(y)^{\frac{\varrho}{1+\varrho}} \mathrm{d} \mu(y) \mathrm{d} \mathrm{Q}(x) \\
& =-(1+\varrho) \log \int_{y \in \mathcal{Y}}\left(\int_{x \in \mathcal{X}} w(y \mid x)^{\frac{1}{1+\varrho}} \mathrm{dQ}(x)\right) \cdot\left(f_{R}(y)^{\frac{\varrho}{1+\varrho}}\right) \mathrm{d} \mu(y) \\
& \geq-\log \int_{y \in \mathcal{Y}}\left(\int_{x \in \mathcal{X}} w(y \mid x)^{\frac{1}{1+\varrho}} \mathrm{dQ}(x)\right)^{1+\varrho} \mathrm{d} \mu(y) \\
& =E_{0}(\varrho, \mathrm{Q}, 0) .
\end{aligned}
$$

As for the case where $\mathrm{E}_{\mathrm{Q}}[g(X)]=\Upsilon$ (and $\left.\mathrm{E}_{\mathrm{Q}}\left[g^{3}(X)\right]<\infty\right)$ we have for 
any $r \geq 0$

$$
\begin{aligned}
&-(1+\varrho) \int_{x \in \mathcal{X}} \log \left(\int_{y \in \mathcal{Y}} w(y \mid x)^{\frac{1}{1+\varrho}} f_{R}(y)^{\frac{\varrho}{1+\varrho}} \mathrm{d} \mu(y)\right) \mathrm{dQ}(x) \\
&=\quad r(1+\varrho)\left(\mathrm{E}_{\mathrm{Q}}[g(X)]-\Upsilon\right) \\
&-(1+\varrho) \int_{x \in \mathcal{X}} \log \left(\int_{y \in \mathcal{Y}} e^{r(g(x)-\Upsilon)} w(y \mid x)^{\frac{1}{1+\varrho}} f_{R}(y)^{\frac{\varrho}{1+\varrho}} \mathrm{d} \mu(y)\right) \mathrm{dQ}(x) \\
&=-(1+\varrho) \int_{x \in \mathcal{X}} \log \left(\int_{y \in \mathcal{Y}} e^{r(g(x)-\Upsilon)} w(y \mid x)^{\frac{1}{1+\varrho}} f_{R}(y)^{\frac{\varrho}{1+\varrho}} \mathrm{d} \mu(y)\right) \mathrm{dQ}(x) \\
& \geq-(1+\varrho) \log \int_{x \in \mathcal{X}} \int_{y \in \mathcal{Y}} e^{r(g(x)-\Upsilon)} w(y \mid x)^{\frac{1}{1+\varrho}} f_{R}(y)^{\frac{\varrho}{1+\varrho}} \mathrm{d} \mu(y) \mathrm{dQ}(x) \\
& \geq-\log \int_{y \in \mathcal{Y}}\left(\int_{x \in \mathcal{X}} e^{r(g(x)-\Upsilon)} w(y \mid x)^{\frac{1}{1+\varrho}} \mathrm{dQ}(x)\right)^{1+\varrho} \mathrm{d} \mu(y) \\
&= E_{0}(\varrho, \mathrm{Q}, r) .
\end{aligned}
$$

where the second equality follows because in the case we are considering now $\mathrm{E}_{\mathbf{Q}}[g(X)]=\Upsilon$; the first inequality by Jensen's inequality, and the subsequent by Hölder's inequality. The result for this case now follows because $r \geq 0$ in the above is arbitrary.

To conclude, to derive lower bounds on $E_{0}(\varrho, \Upsilon)$ we can choose any input distribution $\mathrm{Q}$ satisfying the constraint $\mathrm{E}_{\mathrm{Q}}[g(X)] \leq \Upsilon$ to obtain the lower bound:

$$
E_{0}(\varrho, \Upsilon) \geq E_{\mathrm{G}, 0}^{\mathrm{M}}(\varrho, \mathrm{Q})
$$

where $E_{\mathrm{G}, 0}^{\mathrm{M}}(\varrho, \mathrm{Q})$ is defined in (154).

To derive upper bounds on $E_{0}(\varrho, \Upsilon)$ we can use the above proposition by choosing some arbitrary output density $f_{R}(y)$ to obtain

$$
\begin{aligned}
& E_{0}(\varrho, \Upsilon) \leq \\
& \sup _{\mathbf{Q}: \mathrm{E}_{\mathbf{Q}}[g(X)] \leq \Upsilon}\left\{-(1+\varrho) \int_{x \in \mathcal{X}} \log \left(\int_{y \in \mathcal{Y}} w(y \mid x)^{\frac{1}{1+\varrho}} f_{R}(y)^{\frac{\varrho}{1+\varrho}} \mathrm{d} \mu(y)\right) \mathrm{dQ}(x)\right\} .
\end{aligned}
$$

\section{Ricean Fading Channels}

The discrete-time memoryless Ricean fading channel with partial receiver side information is a channel whose input $x$ takes value in the complex field $\mathbb{C}$ and whose corresponding output constitutes of a pair of complex random variables $Y$ and $S$. We shall refer to $Y$ as "the received signal" and to $S$ 
as the "side information (at the receiver)". The joint distribution of $Y, S$ corresponding to the input $x \in \mathbb{C}$ is best described using the fading complex random variable $H$ and the additive noise complex random variable $Z$.

The joint distribution of $H, S$, and $Z$ does not depend on the input $x$. The additive noise $Z$ is independent of the pair $(H, S)$ and has a circularly symmetric complex Gaussian distribution of positive variance $\sigma^{2}$. The fading $H$ is of mean $d \in \mathbb{C}$ - the "specular component" - and it is assumed that $H-d$ is a unit-variance circularly symmetric complex Gaussian random variable. $^{2}$ The pair $S$ and $H-d$ are jointly circularly symmetric Gaussian random variables. We denote the conditional variance of $H$ given $S$ by $\epsilon^{2}$.

The received signal $Y$ corresponding to the input $x \in \mathbb{C}$ is given by

$$
Y=H x+Z \text {. }
$$

The case where $\epsilon^{2}=1$ corresponds to the case where $H$ and $S$ are independent, in which case the receiver can discard $S$ without loss in information rates. This case corresponds to "non-coherent" fading. In the case $\epsilon^{2}=0$ the receiver can precisely determine the realization of $H$ from $S$. This corresponds to "coherent detection". Finally, the case $0<\epsilon<1$ corresponds to "partially coherent" communication. In this case $S$ carries some information about $H$, but it does not fully determine $H$. In this paper we shall only consider the case where $\epsilon^{2}>0$. The case $\epsilon^{2}=0$ is much easier to analyze and has already received considerable attention in the literature. See for example, 9], [10, [11] and the references in the latter.

The special case of Ricean fading with zero specular component $d$ is called "Rayleigh fading". The non-coherent $\left(\epsilon^{2}=1\right)$ capacity of this channel was studied in [12, [13] and [14. The coherent case $\left(\epsilon^{2}=0\right)$ was studied in 9]. The capacity of the non-coherent Ricean channel $\left(\epsilon^{2}=1\right.$ and $\left.d \neq 0\right)$ was studied in [15]-16] and [14.

Unless some restrictions are imposed on the input $x$, the capacity and cutoff rate of this channel are infinite. Two kinds of restrictions are typically considered. The first corresponds to an average power constraint. Here only blockcodes where each codeword satisfies (32) with

$$
g(x)=|x|^{2}
$$

\footnotetext{
${ }^{2}$ We shall sometimes refer to such Ricean fading as "normalized Ricean fading" to make it explicit that the fading is of unit variance. "Un-normalized" Ricean fading need not have unit-variance. Those can be normalized by scaling the fading and absorbing the scaling into the input power. Note also that there is no loss in generality in assuming that $d$ is real and non-negative. The more general complex case can be treated by rotating the output.
} 
are allowed. In this context rather than denoting the allowed cost by $\Upsilon$ we shall use the more common symbol $\mathcal{E}$, which stands here for the average energy per symbol. That is, we only allow blocklength- $n$ codes in which every codeword $x_{1}, \ldots, x_{n}$ satisfies

$$
\frac{1}{n} \sum_{\ell=1}^{n}\left|x_{\ell}\right|^{2} \leq \mathcal{E} .
$$

The second type of constraint is a peak power constraint. Here we only allow channel inputs that satisfy

$$
|x|^{2} \leq \mathcal{E}
$$

where $\mathcal{E}$ now stands for the allowed peak power. Such a constraint is best treated by considering the channel as being free of constraints but with the input alphabet now being $\left\{z \in \mathbb{C}:|z|^{2} \leq \mathcal{E}\right\}$.

For both the average and peak power constraints we define the signal-tonoise ratio $(\mathrm{SNR})$ as

$$
\mathrm{SNR} \triangleq \frac{\mathcal{E}}{\sigma^{2}}
$$

Any codebook satisfying the peak power constraint (64) also satisfies the average power constraint hence the capacity and reliability function under the peak constraint cannot exceed those under the average constraint.

Irrespective of whether an average power or a peak power constraint is imposed, at high SNR the capacity $C(\mathrm{SNR} \mid S)$ of this channel is given asymptotically as

$$
C(\mathrm{SNR} \mid S)=\log \log \mathrm{SNR}+\log |d|^{2}-\operatorname{Ei}\left(-|d|^{2}\right)-1+\log \frac{1}{\epsilon^{2}}+o(1)
$$

where the correction term $o(1)$ depends on the SNR and tends to zero as the SNR tends to infinity. Here $\operatorname{Ei}(\cdot)$ denotes the Exponential Integral function

$$
\operatorname{Ei}(-\xi)=-\int_{\xi}^{\infty} \frac{e^{-t}}{t} \mathrm{~d} t, \quad \xi>0
$$

and we define the value of the function $\log (\xi)-\operatorname{Ei}(-\xi)$ at $\xi=0$ as $-\gamma$, where $\gamma \approx 0.577$ denotes Euler's constant. (With this definition the function $\log (\xi)-\operatorname{Ei}(-\xi)$ is continuous from the right at $\xi=0$.)

Here we shall study the cutoff rate in two cases. First, in the absence of side information $\left(\epsilon^{2}=1\right)$ we will show that irrespective of whether a peak or average power constraint is imposed

$$
R_{0}(\mathrm{SNR})=\log \log \mathrm{SNR}+\frac{|d|^{2}}{2}-\log (2 \pi)-2 \log \mathrm{I}_{0}\left(\frac{|d|^{2}}{4}\right)+o(1) .
$$




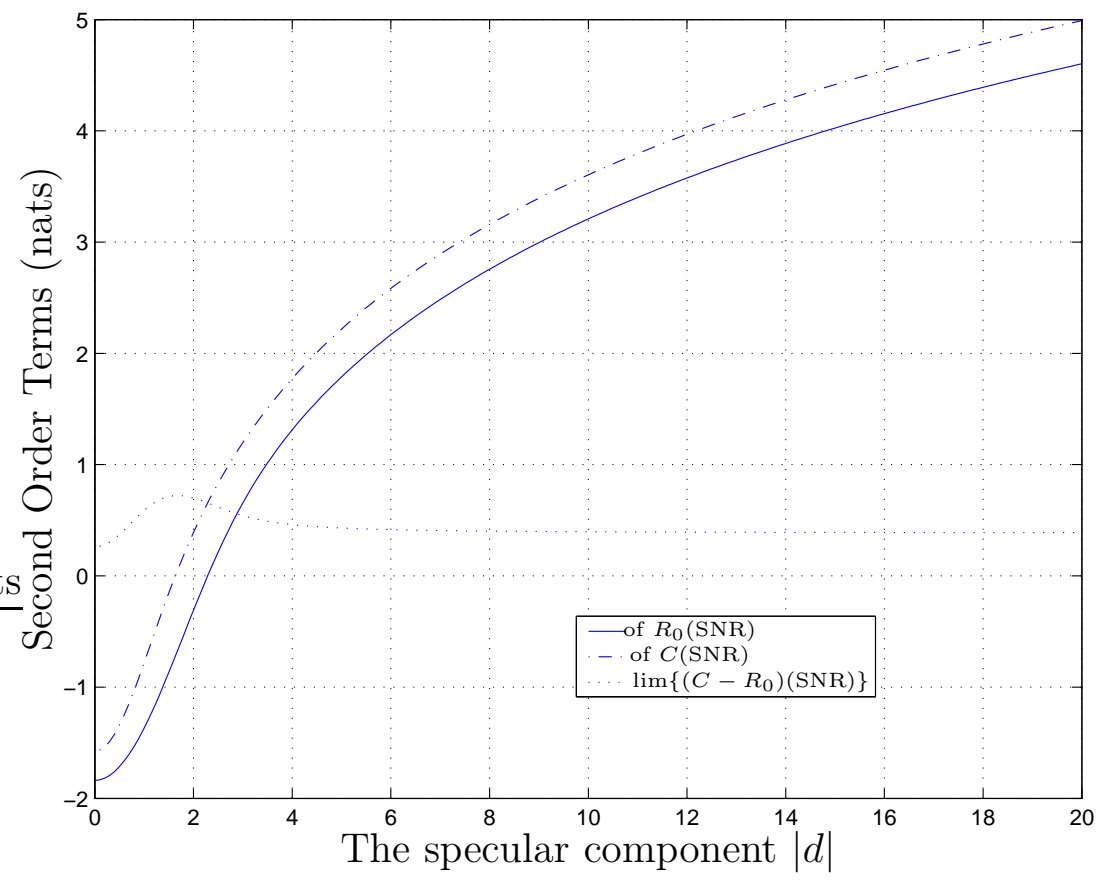

Figure 1: The second order terms of $C(\mathrm{SNR})$ and $R_{0}(\mathrm{SNR})$ and their difference as functions of the specular component $|d|$ for $\epsilon=1$, i.e., in the absence of side information. Upper curve depicts $\lim _{\mathrm{SNR} \rightarrow \infty}\{C(\mathrm{SNR})-\log \log \mathrm{SNR}\}$, followed by the analogous term for the cutoff rate and their difference.

Here $\mathrm{I}_{0}(\cdot)$ denotes the zero-th order modified Bessel function of the first kind, which is given by

$$
\mathrm{I}_{0}(\xi)=\frac{1}{2 \pi} \int_{-\pi}^{\pi} e^{\xi \cos \theta} \mathrm{d} \theta, \quad \xi \in \mathbb{R}
$$

and the $o(1)$ term is a correction term that depends on the SNR and that approaches zero as the SNR tends to infinity.

Figure 1 depicts the second order term (the constant term) in the high SNR expansion of channel capacity (66) and of the cutoff rate (68) as a function of the specular component $d$ in the absence of side information. For a zero specular component the difference between the two second order terms is $\log (2 \pi)-1-\gamma \approx 0.26$ nats; for very large specular components $(|d| \rightarrow \infty)$ this difference approaches $\log (4 / e) \approx 0.39$ nats. $^{3}$

For the case where the side information is present but is not perfect $\left(0<\epsilon^{2}<1\right)$ we only treat the case of zero specular component $(d=0$, i.e.,

\footnotetext{
${ }^{3}$ All logarithms in this paper are natural logarithms.
} 
Rayleigh fading). We obtain the expansion

$$
\begin{array}{r}
R_{0}(\mathrm{SNR} \mid S)=\log \log \mathrm{SNR}+\log \frac{1}{\epsilon^{2}}-\log \mathrm{K}\left(\sqrt{1-\epsilon^{4}}\right)-\log 4+o(1) \\
0<\epsilon^{2}<1, \quad d=0
\end{array}
$$

where $\mathrm{K}(\cdot)$ is the complete elliptic integral of the first kind:

$$
\mathrm{K}(\xi)=\int_{0}^{1} \frac{1}{\sqrt{1-t^{2}} \sqrt{1-\xi^{2} t^{2}}} \mathrm{~d} t, \quad \xi^{2}<1 .
$$

For the case of Rayleigh fading with perfect side information $\left(\epsilon^{2}=0\right)$ see [10]. For the case of "almost perfect side information" $\left(0<\epsilon^{2} \ll 1\right)$ we note the expansion

$$
\begin{array}{r}
\log \frac{1}{\epsilon^{2}}-\log K\left(\sqrt{1-\epsilon^{4}}\right)-\log 4=\log \frac{1}{\epsilon^{2}}-\log \log \frac{4}{\epsilon^{2}}-\log 4+o\left(\epsilon^{4}\right) \\
0<\epsilon^{2} \ll 1 .
\end{array}
$$

which follows from the approximation [17]

$$
\mathrm{K}(k)=\frac{1}{1-\theta} \log \frac{4}{\sqrt{1-k^{2}}}, \quad 0 \leq k<1
$$

for some

$$
0<\theta<\frac{1-k^{2}}{4}
$$

Figure 2 depicts the second order terms of channel capacity (66) and the cutoff rate (70) as a function of the estimation error $\epsilon^{2}$ in estimating the fading from the side information for Rayleigh fading channels $(d=0)$.

\section{Derivations for Ricean Channels}

\subsection{The Cut-Off Rate in Absence of Side Information}

\subsubsection{Upper Bound}

To derive an upper bound on the cut-off rate of the Ricean channel in the absence of side information we use Proposition 1 with the density (w.r.t. the Lebesgue measure $\mu$ on $\mathbb{C}$ )

$$
f_{R}(y)=\frac{\left(|y|^{2}+\delta\right)^{\alpha-1} e^{-\frac{|y|^{2}+\delta}{\beta}}}{\pi \beta^{\alpha} \Gamma(\alpha, \delta / \beta)}, y \in \mathbb{C} .
$$




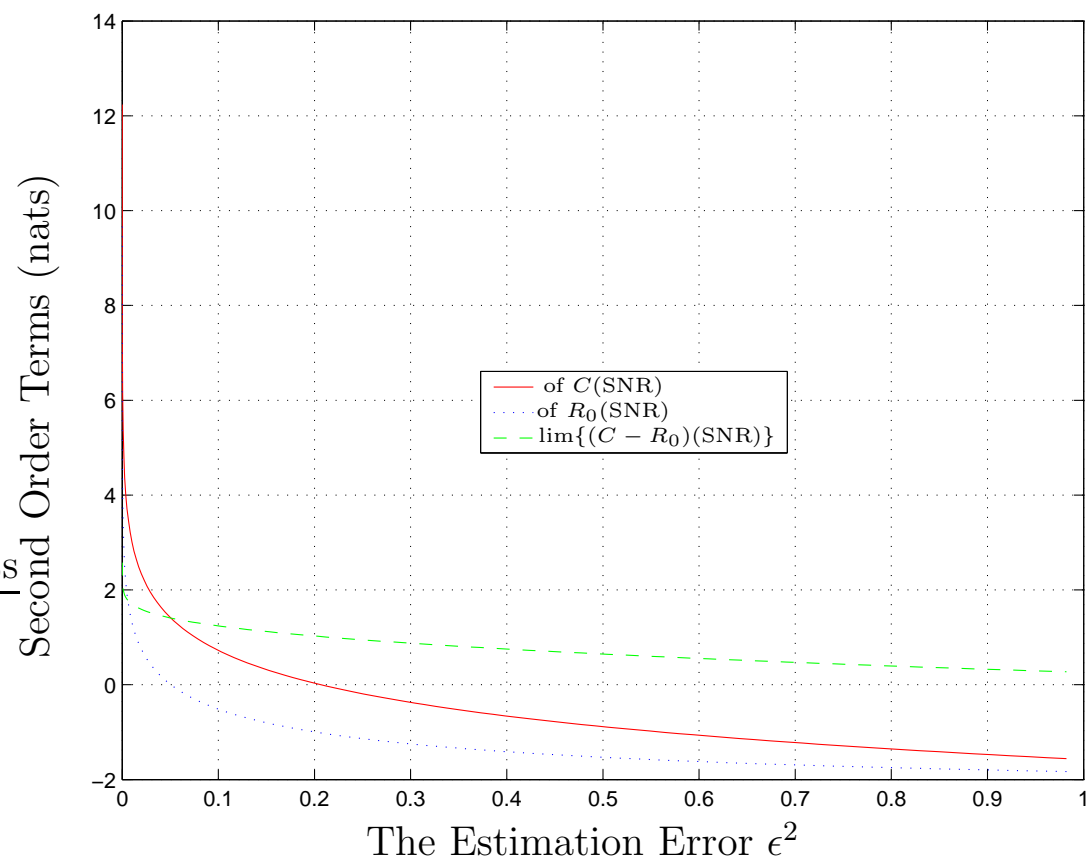

Figure 2: The second order terms of $C(\mathrm{SNR}), R_{0}(\mathrm{SNR})$ and their difference as functions of the minimum mean squared error $\epsilon^{2}$ in estimating the fading from the side information. Rayleigh fading $(d=0)$ is assumed. 
Here the parameters $\delta \geq 0, \alpha>0$, and $\beta>0$ can be chosen freely in order to obtain the tightest bound, and $\Gamma(\alpha, \xi)$ denotes the incomplete Gamma function,

$$
\Gamma(\alpha, \xi)=\int_{\xi}^{\infty} t^{\alpha-1} e^{-t} \mathrm{~d} t, \alpha>0, \xi \geq 0 .
$$

(This family of densities was introduced in 14 for the purpose of studying the fading number.)

By Proposition 1 applied with $\varrho=1$ we obtain for any law $\mathrm{Q}$ under which

$$
\mathrm{E}_{\mathrm{Q}}\left[|X|^{2}\right] \leq \mathcal{E}
$$

the upper bound

$$
E_{\mathrm{G}, 0}^{\mathrm{M}}(1, \mathrm{Q}) \leq-2 \int_{x \in \mathbb{C}} \log \psi(x) \mathrm{dQ}(x)
$$

where

$$
\begin{aligned}
\psi(x) & \triangleq \int_{y \in \mathbb{C}} \sqrt{w(y \mid x) \cdot f_{R}(y)} \mathrm{d} \mu(y) \\
& =\frac{2 e^{\frac{-\delta}{2 \beta}} e^{-\frac{|d|^{2}|x|^{2}}{2\left(|x|^{2}+\sigma^{2}\right)}}}{\sqrt{\Gamma\left(\alpha, \frac{\delta}{\beta}\right)} \beta^{\frac{\alpha}{2}} \sqrt{|x|^{2}+\sigma^{2}}} \ell(x ; \alpha, \beta, \delta)
\end{aligned}
$$

and from [18, 3.338]

$$
\ell(x ; \alpha, \beta, \delta)=\int_{0}^{\infty} e^{-\frac{\rho^{2}\left(\beta+|x|^{2}+\sigma^{2}\right)}{2 \beta\left(|x|^{2}+\sigma^{2}\right)}} \rho\left(\rho^{2}+\delta\right)^{\frac{\alpha-1}{2}} \mathrm{I}_{0}\left(\frac{|d| \cdot|x| \cdot \rho}{|x|^{2}+\sigma^{2}}\right) \mathrm{d} \rho .
$$

For our high SNR analysis it will suffice to consider (for sufficiently large powers $\mathcal{E}$ ) the possibly sub-optimal choice of the parameters

$$
\beta=\mathcal{E} \log \mathcal{E} \quad \alpha=\frac{\delta}{\log \beta}
$$

and to consider the limiting behavior of the bound as $\mathcal{E} \rightarrow \infty$. After taking this limit with $\delta>0$ held fixed we shall consider the additional limit of $\delta \rightarrow 0$.

The analytic computation of $\ell(x ; \alpha, \beta, \delta)$ is difficult. Note, however, that any lower bound to this quantity will yield an upper bound on $E_{\mathrm{G}, 0}^{\mathrm{M}}(1, \mathrm{Q})$. Also, the integral is computable when both $\alpha$ and $\delta$ are formally set to zero. ${ }^{4}$

\footnotetext{
${ }^{4}$ In fact, it suffices that $\delta$ be set to zero.
} 
We can thus use a limiting argument to study $\ell(x ; \alpha, \beta, \delta)$ for $\alpha, \delta$ very small. Indeed, in Appendix $\mathrm{C}$ it is shown that

$$
\ell(x ; \alpha, \beta, \delta) \geq a\left(\alpha, \beta, \delta, m_{1}\right) \cdot \ell(x ; \alpha=0, \beta, \delta=0)
$$

where

$$
a\left(\alpha, \beta, \delta, m_{1}\right)=\delta^{\alpha / 2} \sqrt{\frac{m_{1}}{m_{1}+1}}\left(1-\frac{\sqrt{m_{1} \delta} \cdot \mathrm{I}_{0}\left(\frac{|d| \sqrt{m_{1} \delta}}{2 \sigma}\right)}{\sqrt{\frac{\pi \beta \sigma^{2}}{2\left(\beta+\sigma^{2}\right)}}}\right)
$$

$m_{1}>0$ being some constant. As we shall see, the term $a\left(\alpha, \beta, \delta, m_{1}\right)$ will have a negligible asymptotic contribution to our bound.

The term $\ell(x ; \alpha=0, \beta, \delta=0)$ can be computed analytically [18, 6.618]:

$$
\begin{aligned}
\ell(x ; \alpha=0, \beta, \delta= & 0)=\sqrt{\frac{\pi}{2}} \sqrt{\frac{\beta\left(|x|^{2}+\sigma^{2}\right)}{\beta+|x|^{2}+\sigma^{2}}} \\
& \cdot e^{\frac{\beta|d|^{2}|x|^{2}}{4\left(|x|^{2}+\sigma^{2}\right)\left(\beta+|x|^{2}+\sigma^{2}\right)}} \mathrm{I}_{0}\left(\frac{\beta|d|^{2}|x|^{2}}{4\left(|x|^{2}+\sigma^{2}\right)\left(\beta+|x|^{2}+\sigma^{2}\right)}\right) .
\end{aligned}
$$

We thus conclude from (78) , (80), (83), and (85)

$$
\begin{aligned}
& E_{\mathrm{G}, 0}^{\mathrm{M}}(1, \mathrm{Q}) \leq \frac{\delta}{\beta}-2 \log a\left(\alpha, \beta, \delta, m_{1}\right)+\alpha \log \beta \\
& +\log \Gamma\left(\alpha, \frac{\delta}{\beta}\right)-\log (2 \pi) \\
& \quad+\mathrm{E}_{\mathrm{Q}}\left[\log \left(1+\frac{|X|^{2}+\sigma^{2}}{\beta}\right)\right] \\
& \quad+|d|^{2} \mathrm{E}_{\mathrm{Q}}\left[\frac{|X|^{2}}{|X|^{2}+\sigma^{2}} \cdot\left(1-\frac{\beta}{\beta+|X|^{2}+\sigma^{2}}\right)\right] \\
& \quad+\mathrm{E}_{\mathrm{Q}}\left[\frac{|d|^{2}}{2} \frac{|X|^{2}}{|X|^{2}+\sigma^{2}} \frac{\beta}{\beta+|X|^{2}+\sigma^{2}}-2 \log \mathrm{I}_{0}\left(\frac{|d|^{2}}{4} \frac{|X|^{2}}{|X|^{2}+\sigma^{2}} \frac{\beta}{\beta+|X|^{2}+\sigma^{2}}\right)\right]
\end{aligned}
$$

The expectations in the above cannot be computed without knowledge of the law Q. We thus proceed to upper bound the expectations using the average power constraint (177). The expectation of the logarithm is upper bounded using Jensen's inequality and the power constraint (177); the following expectation is upper bounded using the point-wise upper bound $|x|^{2} /\left(|x|^{2}+\sigma^{2}\right)<1$, Jensen's inequality, and the power constraint (77); and 
the final expectation by noting that the function $\xi \mapsto \xi-2 \log \mathrm{I}_{0}(\xi / 2)$ is monotonically increasing and by noting that

$$
\frac{|d|^{2}}{2} \frac{|X|^{2}}{|X|^{2}+\sigma^{2}} \frac{\beta}{\beta+|X|^{2}+\sigma^{2}}<\frac{|d|^{2}}{2} .
$$

We thus conclude that with the allowed average power $\mathcal{E}$ the cut-off rate satisfies:

$$
\begin{aligned}
R_{0}(\mathcal{E})-\log \log \frac{\mathcal{E}}{\sigma^{2}} \leq & \frac{\delta}{\beta}-2 \log a\left(\alpha, \beta, \delta, m_{1}\right)+\alpha \log \beta \\
& +\log \Gamma\left(\alpha, \frac{\delta}{\beta}\right)-\log \log \frac{\mathcal{E}}{\sigma^{2}} \\
& +\log \left(1+\frac{\mathcal{E}+\sigma^{2}}{\beta}\right) \\
& +\left(1-\frac{\beta}{\beta+\mathcal{E}+\sigma^{2}}\right)|d|^{2} \\
& +\frac{|d|^{2}}{2}-2 \log \mathrm{I}_{0}\left(\frac{|d|^{2}}{4}\right)-\log (2 \pi)
\end{aligned}
$$

Holding $\delta>0$ (small) and $m_{1}>0$ (large) fixed, and letting $\mathcal{E} \rightarrow \infty$ with $\alpha=\alpha(\mathcal{E})$ and $\beta=\beta(\mathcal{E})$ as in (82) we obtain from the above and (84)

$$
\begin{aligned}
\varlimsup_{\mathcal{E} \rightarrow \infty}\left\{R_{0}(\mathcal{E})-\log \log \frac{\mathcal{E}}{\sigma^{2}}\right\} \leq & \log \left(\frac{m_{1}+1}{m_{1}}\right)-2 \log \left(1-\frac{\sqrt{m_{1} \delta} \cdot \mathrm{I}_{0}\left(\frac{|d| \sqrt{m_{1} \delta}}{2 \sigma}\right)}{\sqrt{\frac{\pi \sigma^{2}}{2}}}\right) \\
& +\log \frac{1-e^{-\delta}}{\delta} \\
& +\frac{|d|^{2}}{2}-2 \log \mathrm{I}_{0}\left(\frac{|d|^{2}}{4}\right)-\log (2 \pi)
\end{aligned}
$$

where in computing the limiting difference between the Incomplete Gamma function and $\log \log \mathcal{E}$ we used [14, Appendix XI]. Holding $m_{1}$ fixed and letting $\delta \rightarrow 0$ we obtain

$$
\begin{aligned}
\varlimsup_{\mathcal{E} \rightarrow \infty}\left\{R_{0}(\mathcal{E})-\log \log \frac{\mathcal{E}}{\sigma^{2}}\right\} \leq & \log \left(\frac{m_{1}+1}{m_{1}}\right) \\
& +\frac{|d|^{2}}{2}-2 \log \mathrm{I}_{0}\left(\frac{|d|^{2}}{4}\right)-\log (2 \pi) .
\end{aligned}
$$


Letting now $m_{1}$ tend to infinity we obtain the desired asymptotic upper bound

$$
\varlimsup_{\mathcal{E} \rightarrow \infty}\left\{R_{0}(\mathcal{E})-\log \log \frac{\mathcal{E}}{\sigma^{2}}\right\} \leq \frac{|d|^{2}}{2}-2 \log \mathrm{I}_{0}\left(\frac{|d|^{2}}{4}\right)-\log (2 \pi) .
$$

\subsubsection{Lower Bound}

Any input distribution satisfying the cost constraint (possibly strictly) induces a lower bound on the cut-off rate (56). Indeed, for any input distribution $\tilde{Q}$ satisfying the cost constraint

$$
\begin{aligned}
R_{0}(\Upsilon) & \geq\left. E_{\mathrm{G}, 0}^{\mathrm{M}}(\varrho, \tilde{\mathrm{Q}})\right|_{\varrho=1} \\
& \geq\left. E_{0}(\varrho, \tilde{\mathrm{Q}}, r)\right|_{\varrho=1, r=0}
\end{aligned}
$$

where the first inequality follows by the definition of the cut-off rate (56) (and holds with equality if $\tilde{Q}$ achieves the cut-off rate) and where the second inequality follows from (54) (and holds with equality if $\tilde{Q}$ satisfies the cost constraint with strict inequality).

We thus proceed to lower bound $E_{0}(1, \tilde{Q}, 0)$ for a law $\tilde{Q}$ of our choice. Under this law, $X$ is a circularly symmetric random variable with

$$
\log |X|^{2} \sim \text { Uniform }(\log \log \mathcal{E}, \log \mathcal{E}) .
$$

The motivation for using this law is that it is known to achieve the asymptotic capacity 14. Moreover, this law also satisfies the peak power constraint $|X|^{2} \leq \mathcal{E}$, so that the lower bound on the cut-off rate we compute will also be valid as a lower bound for the cut-off rate under a peak constraint. Finally, as the next proposition shows, the fact that under $\tilde{Q}$ the input $X$ satisfies, with probability one, $|X| \geq x_{\min }$, where $x_{\min } \rightarrow \infty$ greatly simplifies our analysis. It allows us to asymptotically ignore the additive noise.

Proposition 2. Let $E_{0}(1, \mathrm{Q}, 0)$ denote the function $E_{0}(\rho, \mathrm{Q}, r)$ evaluated at $\rho=1, r=0$ for the input law $\mathbf{Q}$ to the Ricean channel of specular component $d$ and additive noise variance $\sigma^{2}$. Let $E_{0}^{\sigma=0}(1, Q, 0)$ be similarly defined for the Ricean channel with the same specular component but without any additive noise. If under the law $\mathrm{Q}$ the input $X \in \mathbb{C}$ satisfies with probability one

$$
|X| \geq x_{\min }
$$

for some $x_{\min }>0$ then

$$
E_{0}(1, \mathrm{Q}, 0) \geq E_{0}^{\sigma=0}(1, \mathrm{Q}, 0)-O\left(\frac{|d|^{2}+1}{x_{\min }^{2}}\right) .
$$


Proof. For any input probability distribution $\mathrm{Q}$, the term $E_{0}(1, \mathrm{Q}, 0)$ can be expressed

$$
\begin{aligned}
E_{0}(1, \mathrm{Q}, 0) & =-\log \int_{x} \int_{x^{\prime}} \int_{y} \sqrt{w(y \mid x) w\left(y \mid x^{\prime}\right)} \mathrm{d} \mu(y) \mathrm{dQ}\left(x^{\prime}\right) \mathrm{dQ}(x) \\
& =-\log \int_{x} \int_{x^{\prime}} B\left(x, x^{\prime} ; \sigma\right) \mathrm{d} \mathrm{Q}\left(x^{\prime}\right) \mathrm{d} \mathrm{Q}(x)
\end{aligned}
$$

where

$$
B\left(x, x^{\prime} ; \sigma\right) \triangleq \int_{y} \sqrt{w(y \mid x) w\left(y \mid x^{\prime}\right)} \mathrm{d} \mu(y)
$$

and where for the Ricean fading channel with additive noise of variance $\sigma^{2}$

$$
B\left(x, x^{\prime} ; \sigma\right)=\frac{2 \sqrt{|x|^{2}+\sigma^{2}} \sqrt{\left|x^{\prime}\right|^{2}+\sigma^{2}}}{\left|x^{\prime}\right|^{2}+|x|^{2}+2 \sigma^{2}} e^{\frac{-|d|^{2} \cdot\left|x-x^{\prime}\right|^{2}}{2\left(|x|^{2}+\left|x^{\prime}\right|^{2}+2 \sigma^{2}\right)}} .
$$

Comparing $B\left(x, x^{\prime} ; \sigma\right)$ with the corresponding term in the absence of noise $B\left(x, x^{\prime} ; 0\right)$ we obtain

$$
\begin{aligned}
& B\left(x, x^{\prime} ; \sigma\right) \\
& \quad \leq B\left(x, x^{\prime} ; 0\right) \sqrt{1+\sigma^{2} /|x|^{2}} \sqrt{1+\sigma^{2} /\left|x^{\prime}\right|^{2}} e^{|d|^{2} \sigma^{2} \frac{\left|x-x^{\prime}\right|^{2}}{\left(|x|^{2}+\left|x^{\prime}\right|^{2}+2 \sigma^{2}\right)\left(|x|^{2}+\left|x^{\prime}\right|^{2}\right)}} \\
& \quad \leq B\left(x, x^{\prime} ; 0\right) \sqrt{1+\sigma^{2} /|x|^{2}} \sqrt{1+\sigma^{2} /\left|x^{\prime}\right|^{2}} e^{|d|^{2} \sigma^{2} \frac{\left(|x|+\left|x^{\prime}\right|\right)^{2}}{\left(|x|^{2}+\left|x^{\prime}\right|^{2}+2 \sigma^{2}\right)\left(|x|^{2}+\left|x^{\prime}\right|^{2}\right)}}
\end{aligned}
$$

where the last inequality follows by the triangle inequality. It thus follows from (91) and (95) that if under the law $\mathrm{Q}$ the random variable $X$ satisfies with probability one $|X| \geq x_{\min }$ then

$$
\begin{aligned}
& E_{0}(1, \mathrm{Q}, 0) \geq E_{0}^{\sigma=0}(1, \mathrm{Q}, 0) \\
&-\sup _{|x|,\left|x^{\prime}\right| \geq x_{\min }}\left\{\log \sqrt{1+\sigma^{2} /|x|^{2}}+\log \sqrt{1+\sigma^{2} /\left|x^{\prime}\right|^{2}}\right. \\
&\left.\quad+|d|^{2} \sigma^{2} \frac{\left(|x|+\left|x^{\prime}\right|\right)^{2}}{\left(|x|^{2}+\left|x^{\prime}\right|^{2}+2 \sigma^{2}\right)\left(|x|^{2}+\left|x^{\prime}\right|^{2}\right)}\right\} \\
&=E_{0}^{\sigma=0}(1, \mathrm{Q}, 0)-O\left(\left(|d|^{2}+1\right) / x_{\text {min }}^{2}\right) .
\end{aligned}
$$

Using this proposition with the law $\tilde{\mathrm{Q}}$ under which $X$ is distributed according to (89) we obtain that

$$
\varliminf_{\mathcal{E} \rightarrow \infty}\left\{R_{0}(\mathcal{E})-E_{0}^{\sigma=0}(1, \tilde{Q}, 0)\right\} \geq 0 .
$$


Computing $E_{0}^{\sigma=0}(1, \tilde{Q}, 0)$ from (91) and (93) we obtain

$$
\begin{aligned}
E_{0}^{\sigma=0}(1, \tilde{\mathrm{Q}}, 0)=- & \log 8+\frac{|d|^{2}}{2}+2 \log \log \frac{\mathcal{E}}{\log \mathcal{E}} \\
& -\log \int_{\sqrt{\log \mathcal{E}}}^{\sqrt{\mathcal{E}}} \int_{\sqrt{\log \mathcal{E}}}^{\sqrt{\mathcal{E}}} \frac{1}{\rho^{2}+\rho^{\prime 2}} \mathrm{I}_{0}\left(\frac{|d|^{2} \rho \rho^{\prime}}{\rho^{2}+\rho^{\prime 2}}\right) \mathrm{d} \rho \mathrm{d} \rho^{\prime} .
\end{aligned}
$$

The last term on the RHS of the above is difficult to evaluate precisely. However, since the integrand is positive, the double integral can be upper bounded by inflating the region of integration to the region

$$
\left\{\rho, \rho^{\prime} \geq 0: 2 \log \mathcal{E} \leq \rho^{2}+\rho^{\prime 2} \leq 2 \mathcal{E}\right\}
$$

The integral over this larger set can be now computed analytically by changing to polar coordinates to obtain

$$
\int_{\sqrt{\log \mathcal{E}}}^{\sqrt{\mathcal{E}}} \int_{\sqrt{\log \mathcal{E}}}^{\sqrt{\mathcal{E}}} \frac{1}{\rho^{2}+\rho^{\prime 2}} \mathrm{I}_{0}\left(\frac{|d|^{2} \rho \rho^{\prime}}{\rho^{2}+\rho^{\prime 2}}\right) \mathrm{d} \rho \mathrm{d} \rho^{\prime} \leq \frac{\pi}{2} \mathrm{I}_{0}^{2}\left(\frac{|d|^{2}}{4}\right) \cdot \log \sqrt{\frac{\mathcal{E}}{\log \mathcal{E}}}
$$

where we have used the identity

$$
\frac{2}{\pi} \int_{0}^{\frac{\pi}{2}} \mathrm{I}_{0}(\xi \sin \varphi) \mathrm{d} \varphi=\mathrm{I}_{0}^{2}(\xi / 2), \quad \xi \in \mathbb{R}
$$

which follows from [18, 6.567]. Consequently, by (97) and (98)

$$
E_{0}^{\sigma=0}(1, \tilde{Q}, 0) \geq \log \log \frac{\mathcal{E}}{\log \mathcal{E}}+\frac{|d|^{2}}{2}-\log (2 \pi)-2 \log I_{0}\left(\frac{|d|^{2}}{4}\right)
$$

so that by (96)

$$
\varliminf_{\mathcal{E} \rightarrow \infty}\left\{R_{0}(\mathcal{E})-\log \log \frac{\mathcal{E}}{\sigma^{2}}\right\} \geq \frac{|d|^{2}}{2}-\log (2 \pi)-2 \log \mathrm{I}_{0}\left(\frac{|d|^{2}}{4}\right) .
$$

\subsection{The Cut-Off Rate in the Presence of Receiver Side Information}

We next consider the case where the fading $H$ is of zero-mean (Rayleigh) and where the receiver has access to some side-information $S$ that is jointly Gaussian with $H$. We assume that the pair $(H, S)$ is independent of the additive noise $Z$ and that the joint law of $(H, S)$ and $Z$ does not depend on 
the channel input $x \in \mathbb{C}$. We denote the conditional mean of $H$ given $S=s$ by

$$
\hat{d}_{s} \triangleq \mathrm{E}[H \mid S=s]
$$

and the estimation error by

$$
\epsilon^{2} \triangleq \mathrm{E}\left[\left|H-\hat{d}_{s}\right|^{2} \mid S=s\right]
$$

Note that unconditionally, $\hat{d}_{s}$ is a zero-mean circularly-symmetric Gaussian random variable of variance $1-\epsilon^{2}$ :

$$
\hat{d}_{s} \sim \mathcal{N}_{\mathbb{C}}\left(0,1-\epsilon^{2}\right) .
$$

Recall also that we only treat here the case $\epsilon^{2}>0$. Denoting the conditional density of $(Y, S)$ corresponding to the input $x \in \mathbb{C}$ by $w(y, s \mid x)$, we have by the independence of the side information $S$ and the input that

$$
w(y, s \mid x)=f_{S}(s) w(y \mid x, s)
$$

where $f_{S}$ is the density of the side information and where $w(y \mid x, s)$ is the conditional law of $Y$ given the input $x$ and the side information $s$. Note that, because $(H, S)$ are jointly Gaussian, the density $w(y \mid x, s)$ is the Gaussian density of mean $\hat{d}_{s} \cdot x$ and variance $\epsilon^{2} \cdot|x|^{2}+\sigma^{2}$. Consequently,

$$
\begin{aligned}
& E_{0}(1, \mathrm{Q}, r) \\
& =-\log \int_{y} \int_{s}\left(\int_{x} e^{r\left(|x|^{2}-\mathcal{E}\right)} \sqrt{w(y, s \mid x)} \mathrm{dQ}(x)\right)^{2} \mathrm{~d} \mu(y) \mathrm{d} \mu(s) \\
& \quad=-\log \int_{s} f_{S}(s) \int_{y}\left(\int_{x} e^{r\left(|x|^{2}-\mathcal{E}\right)} \sqrt{w(y \mid x, s)} \mathrm{dQ}(x)\right)^{2} \mathrm{~d} \mu(y) \mathrm{d} \mu(s) \\
& =-\log \int_{s} f_{S}(s) \cdot \operatorname{Exp}\left(-E_{0}(1, \mathrm{Q}, r \mid s)\right) \mathrm{d} s
\end{aligned}
$$

where (106) follows from (105) and where (107) follows by defining

$$
E_{0}(1, \mathrm{Q}, r \mid s) \triangleq-\log \int_{y}\left(\int_{x} e^{r\left(|x|^{2}-\mathcal{E}\right)} \sqrt{w(y \mid x, s)} \mathrm{dQ}(x)\right)^{2} \mathrm{~d} \mu(y)
$$

as the $E_{0}$ function corresponding to the channel $w(y \mid x, s)$ for $S=s$ fixed. (This channel is a Ricean fading channel, except that the fading is not normalized to have unit variance.) 
The cut-off rate $R_{0}(\mathcal{E} \mid S)$ in the presence of the side information $S$ can be thus upper bounded by

$$
\begin{aligned}
R_{0}(\mathcal{E} \mid S) & \leq \sup _{\mathrm{Q}: E_{Q}\left[|X|^{2}\right] \leq \mathcal{E}} \sup _{r \geq 0}\left\{-\log \int_{s} f_{S}(s) \cdot \operatorname{Exp}\left(-E_{0}(1, \mathrm{Q}, r \mid s)\right) \mathrm{d} s\right\} \\
& \leq-\log \int_{s} f_{S}(s) \cdot \operatorname{Exp}\left(-\sup _{\left.\mathrm{Q}:\left.E_{Q}|| X\right|^{2}\right] \leq \mathcal{E}} \sup _{r \geq 0} E_{0}(1, \mathrm{Q}, r \mid s)\right) \mathrm{d} s \\
& =-\log \int_{s} f_{S}(s) \cdot \operatorname{Exp}\left(-R_{0}(\mathcal{E} \mid S=s)\right)
\end{aligned}
$$

where

$$
R_{0}(\mathcal{E} \mid S=s) \triangleq \sup _{\left.\mathrm{Q}:\left.\mathrm{E}_{\mathbf{Q}}|| X\right|^{2}\right] \leq \mathcal{E}} \sup _{r \geq 0} E_{0}(1, \mathrm{Q}, r \mid s)
$$

is the cut-off rate corresponding to power $\mathcal{E}$ communication over the channel $w(y \mid x, s)$ for fixed $S=s .{ }^{5}$

It now follows from (111) that

$$
R_{0}(\mathcal{E} \mid S)-\log \log \frac{\mathcal{E}}{\sigma^{2}} \leq-\log \int_{s} f_{S}(s) \cdot \operatorname{Exp}\left(-\left(R_{0}(\mathcal{E} \mid S=s)-\log \log \frac{\mathcal{E}}{\sigma^{2}}\right)\right)
$$

and consequently

$$
\begin{aligned}
\varlimsup_{\mathcal{E} \rightarrow \infty} & \left\{R_{0}(\mathcal{E} \mid S)-\log \log \frac{\mathcal{E}}{\sigma^{2}}\right\} \\
& \leq \varlimsup_{\mathcal{E} \rightarrow \infty}\left\{-\log \int_{s} f_{S}(s) \cdot \operatorname{Exp}\left(-\left(R_{0}(\mathcal{E} \mid S=s)-\log \log \frac{\mathcal{E}}{\sigma^{2}}\right)\right) \mathrm{d} s\right\} \\
& =-\log \frac{\lim }{\mathcal{E} \rightarrow \infty} \int_{s} f_{S}(s) \cdot \operatorname{Exp}\left(-\left(R_{0}(\mathcal{E} \mid S=s)-\log \log \frac{\mathcal{E}}{\sigma^{2}}\right)\right) \mathrm{d} s \\
& \leq-\log \int_{s} f_{S}(s) \underline{\varliminf_{\mathcal{E} \rightarrow \infty}} \operatorname{Exp}\left(-\left(R_{0}(\mathcal{E} \mid S=s)-\log \log \frac{\mathcal{E}}{\sigma^{2}}\right)\right) \mathrm{d} s \\
& =-\log \int_{s} f_{S}(s) \cdot \operatorname{Exp}\left(-\lim _{\mathcal{E} \rightarrow \infty}\left\{R_{0}(\mathcal{E} \mid S=s)-\log \log \frac{\mathcal{E}}{\sigma^{2}}\right\}\right) \mathrm{d} s \\
& =-\log \int_{s} f_{S}(s) \cdot \operatorname{Exp}\left(-\frac{\left|\hat{d}_{s}\right|^{2}}{2 \epsilon^{2}}+\log (2 \pi)+2 \log \mathrm{I}_{0}\left(\frac{\left|\hat{d}_{s}\right|^{2}}{4 \epsilon^{2}}\right)\right) \mathrm{d} s \\
& =\log \frac{1}{\epsilon^{2}}-\log \mathrm{K}\left(\sqrt{1-\epsilon^{4}}\right)-\log 4 .
\end{aligned}
$$

\footnotetext{
${ }^{5}$ This definition is consistent with (55) since the cost constraint on the cut-off rate is always active for the Ricean fading channel.
} 
Here the swapping of the limit and the expectation (second inequality) is justified using Fatou's lemma and we use the result

$$
\lim _{\mathcal{E} \rightarrow \infty}\left\{R_{0}(\mathcal{E} \mid S=s)-\log \log \frac{\mathcal{E}}{\sigma^{2}}\right\}=\frac{\left|\hat{d}_{s}\right|^{2}}{2 \epsilon^{2}}-\log (2 \pi)-2 \log \mathrm{I}_{0}\left(\frac{\left|\hat{d}_{s}\right|^{2}}{4 \epsilon^{2}}\right)
$$

which follows from (68) applied to the un-normalized Ricean fading channel whose specular component is $\hat{d}_{s}$ and whose granular component is of variance $\epsilon^{2}$. The evaluation of the last integral is based on an identity combining [18, $6.612]$ and [19, 160.02]

$$
\int_{0}^{\infty} e^{-\alpha x}\left(\mathrm{I}_{0}(\beta x)\right)^{2} \mathrm{~d} x=\frac{2}{\pi \alpha} K\left(\frac{2 \beta}{\alpha}\right), \quad \alpha, \beta>0
$$

and the identity for the elliptic function [20, Eq. (3.2.4)]

$$
K(k)=\frac{2}{1+k^{\prime}} K\left(\frac{1-k^{\prime}}{1+k^{\prime}}\right), \quad k^{2}+k^{\prime 2}=1,0<k, k^{\prime}<1 .
$$

In view of (118), to establish (70) it now suffices to show

$$
\varliminf_{\mathcal{E} \rightarrow \infty}\left\{R_{0}(\mathcal{E} \mid S)-\log \log \frac{\mathcal{E}}{\sigma^{2}}\right\} \geq \log \frac{1}{\epsilon^{2}}-\log \mathrm{K}\left(\sqrt{1-\epsilon^{4}}\right)-\log 4 .
$$

To this end we note that by (107) and (108) evaluated at $r=0$

$$
R_{0}(\mathcal{E} \mid S) \geq-\log \int_{s} f_{S}(s) \cdot \operatorname{Exp}\left(-E_{0}(1, \tilde{\mathrm{Q}}, 0 \mid s)\right) \mathrm{d} s
$$

for any law $\tilde{\mathrm{Q}}$ satisfying $\mathrm{E}_{\tilde{\mathrm{Q}}}\left[|X|^{2}\right] \leq \mathcal{E}$. We next choose, as before, $\tilde{\mathrm{Q}}$ to be a law under which $X$ is circularly symmetric with

$$
\log |X|^{2} \sim \text { Uniform }(\log \log \mathcal{E}, \log \mathcal{E})
$$

whence by Proposition 2 and (100) applied to the Ricean channel of fading mean $\hat{d}_{s}$ and granular component $\epsilon^{2}$ and the tightness of the lower bound

$$
\lim _{\mathcal{E} \rightarrow \infty}\left\{E_{0}(1, \tilde{Q}, 0 \mid s)-\log \log \frac{\mathcal{E}}{\sigma^{2}}\right\}=\frac{\left|d_{s}\right|^{2}}{2 \epsilon^{2}}-\log (2 \pi)-2 \log \mathrm{I}_{0}\left(\frac{\left|d_{s}\right|^{2}}{4 \epsilon^{2}}\right)
$$

for every $s$. The desired result (121) now follows from (122) and (124) using the Dominated Convergence Theorem and (104). 


\section{A A Lagrange Duality}

In this appendix we prove the following Lagrange duality:

Proposition 3. For any discrete memoryless channel and any $\varrho>0$, the problem

$$
\min _{\mathbf{Q}} e^{-E_{\mathrm{CK}, 0}(\varrho, \mathrm{Q})}
$$

is a Lagrange dual of the problem

$$
\min _{\mathbf{Q}} e^{-E_{\mathrm{G}, 0}(\varrho, \mathrm{Q})}
$$

where $\mathrm{Q}$ is a distribution on the input alphabet. In particular, since strong duality holds,

$$
\max _{\mathbf{Q}} E_{\mathrm{G}, 0}(\varrho, \mathrm{Q})=\max _{\mathrm{Q}} E_{\mathrm{CK}, 0}(\varrho, \mathrm{Q})
$$

Proof. Consider a discrete memoryless channel $\mathrm{W}(y \mid x)$ with input $X \in \mathcal{X}$, $|\mathcal{X}|=N$ and output $Y \in \mathcal{Y},|\mathcal{Y}|=M$. We henceforth introduce the more standard, for optimization problems, vector notation for functions on discrete domains. Hence, let $\mathbf{q} \in \mathbb{R}^{1 \times N}$ be a probability distribution on $\mathcal{X}$ and $\mathbf{w} \in$ $\mathbb{R}^{N \times M}$ be a matrix whose (i,j)-th element is given by

$$
w_{i j}=\mathbf{W}\left(y_{j} \mid x_{i}\right)^{\frac{1}{\varrho+1}}, x_{i} \in \mathcal{X}, y_{j} \in \mathcal{Y}, \varrho>0 .
$$

Hence, (126) can be written as:

$$
\begin{aligned}
& \min _{\mathbf{q}, \mathbf{f}} \sum_{j} f_{j}^{1+\varrho} \\
& \text { s.t. } \mathbf{q} \mathbf{w}=\mathbf{f}, \mathbf{q} \succeq \mathbf{0}, \mathbf{q} \mathbf{1}=1,
\end{aligned}
$$

where $\mathbf{f} \in \mathbb{R}^{1 \times M}$ is an auxiliary vector that we introduce in this problem. The domain $\mathrm{D}$ of this optimization problem is $\mathrm{D}=\{(\mathbf{q}, \mathbf{f}) \mid \mathbf{q} \succeq \mathbf{0}\}$. For any $\varrho>0$ the objective function is convex in D. Furthermore, all equality and inequality constraints are affine. Hence, the problem is a convex optimization problem. We will perform a relaxation, which is nevertheless tight for the optimal values of $\mathbf{f}$ and $\mathbf{q}$, to the constraint $\mathbf{q w}=\mathbf{f}$, namely

$$
\begin{aligned}
& \min _{\mathbf{q}, \mathbf{f}} \sum_{j} f_{j}^{1+\varrho} \\
& \text { s.t. } \mathbf{f} \succeq \mathbf{q w}, \mathbf{q} \succeq \mathbf{0}, \mathbf{q} \mathbf{1}=1 .
\end{aligned}
$$

The Lagrangian function of this problem is

$$
L(\mathbf{q}, \mathbf{f}, \boldsymbol{\nu}, \mu, \boldsymbol{\lambda})=\sum_{j} f_{j}^{1+\varrho}+(\mathbf{q} \mathbf{w}-\mathbf{f}) \boldsymbol{\nu}+(1-\mathbf{q} \mathbf{1}) \mu-\mathbf{q} \boldsymbol{\lambda},
$$


where $\boldsymbol{\lambda} \succeq \mathbf{0} \in \mathbb{R}^{N \times 1}, \boldsymbol{\nu} \succeq \mathbf{0} \in \mathbb{R}^{M \times 1}, \mathbf{f} \in \mathbb{R}^{1 \times M}, \mu \in \mathbb{R}$ and $(\mathbf{q}, \mathbf{f}) \in \mathrm{D}$. Since the Lagrangian function is affine with respect to $\mathbf{q}$, we impose the dual inequality constraint $\mu \mathbf{1} \preceq \mathbf{w} \boldsymbol{\nu}$, minimize the Lagrangian over $\mathbf{f}$ and obtain the Lagrange dual problem

$$
\begin{aligned}
& \max _{\boldsymbol{\nu}, \mu}\left\{-\varrho \sum_{j}\left(\frac{\nu_{j}}{1+\varrho}\right)^{\frac{1+\varrho}{\varrho}}+\mu\right\} \\
& \text { s.t. } \mu \mathbf{1} \preceq \mathbf{w} \boldsymbol{\nu}, \boldsymbol{\nu} \succeq \mathbf{0} .
\end{aligned}
$$

This is a concave problem, with the objective function being monotonic with respect to all the optimization variables. Since we maximize it in a polyhedron, the optimum will be on the boundary, of maximum distance from the hyperplane $\mu=0$ and of minimum distance from all hyperplanes that define the polyhedron. Therefore, some dual constraint has to be active, i.e.,

$$
\min _{i} \sum_{j} w_{i j} \nu_{j}=\mu \text {. }
$$

Consequently, the dual problem becomes

$$
\max _{\boldsymbol{\nu} \succeq \mathbf{0}}\left\{-\varrho \sum_{j}\left(\frac{\nu_{j}}{1+\varrho}\right)^{\frac{1+\varrho}{\varrho}}+\min _{i}\left\{\sum_{j} w_{i j} \nu_{j}\right\}\right\} .
$$

We perform the transformation of variables $\frac{\nu_{j}}{1+\varrho}=r_{j}^{\frac{\varrho}{1+\varrho}} \alpha, j=1, \ldots, M$, where $\mathbf{r} \in \mathbb{R}^{1 \times M}$ is chosen to be a probability distribution and $\alpha \in \mathbb{R}^{+}$is the appropriate normalizing scalar. Optimizing over $\alpha$ yields

$$
\begin{aligned}
& \max _{\mathbf{r}}\left\{\left(\min _{i} \sum_{j} w_{i j} r_{j}^{\frac{\varrho}{\varrho+1}}\right)^{\varrho+1}\right\} \\
& \text { s.t. } \mathbf{r} \succeq \mathbf{0}, \mathbf{r} \mathbf{1}=1
\end{aligned}
$$

which, because of the fact that $\left(\sum_{j} w_{i j} r_{j}^{\frac{\varrho}{\varrho+1}}\right)^{\varrho+1}$ is concave with respect to $\mathbf{r}$ and monotonic with respect to $\sum_{j} w_{i j} r_{j}^{\frac{\varrho}{\varrho+1}}$, concludes the proof.

\section{B Proof of (47)}

Proof. We begin with the case where the cost constraint is active. Fix some $\varrho \geq 0$ and let $\mathrm{Q}_{*}$ and $r_{*}$ achieve

$$
\max _{\mathrm{Q}: \mathrm{E}_{\mathrm{Q}}[g(X)]=\Upsilon} \max _{r \geq 0} E_{0}(\varrho, \mathrm{Q}, r)
$$


so that

$$
E_{0}\left(\varrho, Q_{*}, r_{*}\right)=\max _{Q: E_{Q}[g(X)]=\Upsilon} \max _{r \geq 0} E_{0}(\varrho, Q, r) .
$$

Following [4, Eq. (7.3.26)] we define

$$
\alpha(y) \triangleq \sum_{x \in \mathcal{X}} \mathrm{Q}_{*}(x) e^{r_{*}(g(x)-\Upsilon)} \mathrm{W}(y \mid x)^{\frac{1}{1+\varrho}}, \quad y \in \mathcal{Y} .
$$

With this definition we have by (127) and (36)

$$
\begin{aligned}
\max _{\mathrm{Q}: \mathrm{E}_{\mathrm{Q}}[g(X)]=\Upsilon} \max _{r \geq 0} E_{0}(\varrho, \mathrm{Q}, r) & =E_{0}\left(\varrho, \mathrm{Q}_{*}, r_{*}\right) \\
& =-\log \sum_{y \in \mathcal{Y}} \alpha^{1+\varrho}(y) .
\end{aligned}
$$

Also, by [4, Eq. (7.3.28)]

$$
\sum_{y \in \mathcal{Y}} \alpha^{\varrho}(y) e^{r_{*}(g(x)-\Upsilon)} \mathbf{W}(y \mid x)^{\frac{1}{1+\varrho}} \geq \sum_{y \in \mathcal{Y}} \alpha^{1+\varrho}(y), \quad \forall x \in \mathcal{X} .
$$

Consider now the distribution $\mathrm{R}_{*}$ on $\mathcal{Y}$ given by

$$
\mathrm{R}_{*}(y)=\frac{\alpha^{1+\varrho}(y)}{\sum_{y^{\prime} \in \mathcal{Y}} \alpha^{1+\varrho}\left(y^{\prime}\right)}, \quad y \in \mathcal{Y} .
$$

We now have by (18) that for any distribution $Q$

$$
E_{\mathrm{CK}, 0}(\varrho, \mathrm{Q}) \leq-(1+\varrho) \sum_{x \in \mathcal{X}} \mathrm{Q}(x) \log \left(\sum_{y \in \mathcal{Y}} \mathrm{W}(y \mid x)^{\frac{1}{1+\varrho}} \mathrm{R}_{*}(y)^{\frac{\varrho}{1+\varrho}}\right)
$$

and if $\mathrm{E}_{\mathrm{Q}}[g(X)]=\Upsilon$ then

$$
\begin{aligned}
& E_{\mathrm{CK}, 0}(\varrho, \mathrm{Q}) \\
& \quad \leq-(1+\varrho) \sum_{x \in \mathcal{X}} \mathrm{Q}(x) \log \left(\sum_{y \in \mathcal{Y}} e^{r_{*}(g(x)-\Upsilon)} \mathrm{W}(y \mid x)^{\frac{1}{1+\varrho}} \mathrm{R}_{*}(y)^{\frac{\varrho}{1+\varrho}}\right) \\
& \quad=-(1+\varrho) \sum_{x \in \mathcal{X}} \mathrm{Q}(x) \log \left(\sum_{y \in \mathcal{Y}} e^{r_{*}(g(x)-\Upsilon)} \mathrm{W}(y \mid x)^{\frac{1}{1+\varrho}} \alpha^{\varrho}(y)\right)+\varrho \log \sum_{y \in \mathcal{Y}} \alpha^{1+\varrho}(y) \\
& \quad \leq-\log \sum_{y \in \mathcal{Y}} \alpha^{1+\varrho}(y) \\
& \quad=\max _{\mathrm{Q}: \mathrm{E}_{\mathrm{Q}}[g(X)]=\Upsilon} \max _{r \geq 0} E_{0}(\varrho, \mathrm{Q}, r) .
\end{aligned}
$$


Here the first inequality follows from (132) because the condition $\mathrm{E}_{\mathrm{Q}}[g(X)]=$ $\Upsilon$ guarantees that the introduction of the exponential term $\exp \left\{r_{*}(g(x)-\right.$ $\Upsilon)\}$ has zero net effect; the subsequent equality by (131); the subsequent inequality by (130); and the final equality by (127). It thus follows upon taking the supremum in the above over all laws $\mathrm{Q}$ satisfying $\mathrm{E}_{\mathrm{Q}}[g(X)]=\Upsilon$ that

$$
\max _{\mathrm{Q}: \mathrm{E}_{\mathrm{Q}}[g(X)]=\Upsilon} E_{\mathrm{CK}, 0}(\varrho, \mathrm{Q}) \leq \max _{\mathrm{Q}: \mathrm{E}_{\mathrm{Q}}[g(X)]=\Upsilon} \max _{r \geq 0} E_{0}(\varrho, \mathrm{Q}, r) .
$$

On the other hand, by (46) we obtain

$$
\max _{\mathrm{Q}: \mathrm{E}_{\mathrm{Q}}[g(X)]=\Upsilon} E_{\mathrm{CK}, 0}(\varrho, \mathrm{Q}) \geq \max _{\mathrm{Q}: \mathrm{E}_{\mathrm{Q}}[g(X)]=\Upsilon} \max _{r \geq 0} E_{0}(\varrho, \mathrm{Q}, r)
$$

which combines with (133) to prove the claim for active cost constraints.

For the case of inactive cost constraints we have

$$
\begin{aligned}
\max _{\mathrm{Q}_{\mathrm{E}_{\mathrm{Q}}[g(X)] \leq \Upsilon}} E_{\mathrm{CK}, 0}(\varrho, \mathrm{Q}) & \leq \max _{\mathrm{Q}} E_{\mathrm{CK}, 0}(\varrho, \mathrm{Q}) \\
& =\max _{\mathrm{Q}} E_{\mathrm{G}, 0}(\varrho, \mathrm{Q}) \\
& =\max _{\mathrm{Q}: \mathrm{E}_{\mathrm{Q}}[g(X)] \leq \Upsilon} E_{\mathrm{G}, 0}^{\mathrm{M}}(\varrho, \mathrm{Q}) .
\end{aligned}
$$

Here the first inequality follows by relaxing the constraint; the subsequent equality by (21); and the final equality by (43). This combines with (46) to conclude the proof.

\section{Derivation of (83)}

To derive (83) we begin by noting that for $\rho \geq 1$ the integrand can be lower bounded by its value when $\alpha=0$ because

$$
\left(\rho^{2}+\delta\right)^{\frac{\alpha-1}{2}} \geq\left(\rho^{2}+\delta\right)^{-\frac{1}{2}}, \quad \alpha, \delta \geq 0, \rho \geq 1 .
$$

In the region $0 \leq \rho \leq 1$ we can use the inequality

$$
\left(\rho^{2}+\delta\right)^{\frac{\alpha-1}{2}} \geq \delta^{\frac{\alpha}{2}}\left(\rho^{2}+\delta\right)^{-\frac{1}{2}}, \quad \alpha, \delta>0,0 \leq \rho \leq 1
$$

Combining the above two bounds we obtain that throughout the region of integration

$$
\left(\rho^{2}+\delta\right)^{\frac{\alpha-1}{2}} \geq \delta^{\frac{\alpha}{2}}\left(\rho^{2}+\delta\right)^{-\frac{1}{2}}, \quad \alpha>0,0<\delta<1,0 \leq \rho<\infty
$$

and hence

$$
\ell(x ; \alpha, \beta, \delta) \geq \delta^{\frac{\alpha}{2}} \cdot \ell(x ; \alpha=0, \beta, \delta) .
$$


We next relate $\ell(x ; \alpha=0, \beta, \delta)$ to $\ell(x ; \alpha=0, \beta, \delta=0)$. To that end denote the integrand in $\ell(x ; \alpha=0, \beta, \delta)$ by

$$
\eta(\rho ; x, \beta, \delta, d)=e^{-\rho^{2} \frac{\beta+|x|^{2}+\sigma^{2}}{2 \beta\left(|x|^{2}+\sigma^{2}\right)}} \sqrt{\frac{\rho^{2}}{\rho^{2}+\delta}} \mathrm{I}_{0}\left(\frac{|d| \cdot|x| \cdot \rho}{|x|^{2}+\sigma^{2}}\right) .
$$

We now write the integral as

$$
\ell(x ; \alpha=0, \beta, \delta)=\int_{0}^{\sqrt{m_{1} \delta}}+\int_{\sqrt{m_{1} \delta}}^{\infty} \eta(\rho ; x, \beta, \delta, d) \mathrm{d} \rho .
$$

In the region $\rho \geq \sqrt{m_{1} \delta}$ we have

$$
\sqrt{\frac{\rho^{2}}{\rho^{2}+\delta}} \geq \sqrt{\frac{m_{1}}{m_{1}+1}}
$$

and hence

$$
\int_{\sqrt{m_{1} \delta}}^{\infty} \eta(\rho ; x, \beta, \delta, d) \mathrm{d} \rho \geq \sqrt{\frac{m_{1}}{m_{1}+1}} \int_{\sqrt{m_{1} \delta}}^{\infty} \eta(\rho ; x, \beta, \delta=0, d) \mathrm{d} \rho .
$$

We next show that when $\sqrt{m_{1} \delta}$ is small, the integral over the interval $\left[0, \sqrt{m_{1} \delta}\right]$ is also small. Indeed,

$$
\frac{|x|}{|x|^{2}+\sigma^{2}} \leq \frac{1}{2 \sigma}, \quad x \in \mathbb{C}
$$

which combines with the monotonicity of $\mathrm{I}_{0}(\cdot)$ and the fact that the argument to the exponential function is negative to demonstrate that

$$
0 \leq \eta(\rho ; x, \beta, \delta=0, d) \leq \mathrm{I}_{0}\left(\frac{|d| \cdot \rho}{2 \sigma}\right)
$$

and hence that

$$
0 \leq \int_{0}^{\sqrt{m_{1} \delta}} \eta(\rho ; x, \beta, \delta=0, d) \mathrm{d} \rho \leq \sqrt{m_{1} \delta} \cdot \mathrm{I}_{0}\left(\frac{|d| \sqrt{m_{1} \delta}}{2 \sigma}\right) .
$$

On the other hand a straightforward calculation demonstrates that

$$
\begin{aligned}
\int_{0}^{\infty} \eta(\rho ; x, \beta, \delta=0, d) \mathrm{d} \rho & \geq \int_{0}^{\infty} \eta(\rho ; x, \beta, \delta=0, d=0) \mathrm{d} \rho \\
& =\sqrt{\frac{\pi}{2} \cdot \sqrt{\frac{\beta\left(|x|^{2}+\sigma^{2}\right)}{\beta+|x|^{2}+\sigma^{2}}}} \\
& \geq \sqrt{\frac{\pi \beta \sigma^{2}}{2\left(\beta+\sigma^{2}\right)}}
\end{aligned}
$$


where the first inequality follows from the monotonicity of $\mathrm{I}_{0}(\cdot)$ and the final inequality follows from simple algebra. We thus conclude that

$$
\begin{aligned}
\ell(x ; \alpha=0, \beta, \delta) \\
\quad=\int_{0}^{\infty} \eta(\rho ; x, \beta, \delta, d) \mathrm{d} \rho \\
\geq \int_{\sqrt{m_{1} \delta}}^{\infty} \eta(\rho ; x, \beta, \delta, d) \mathrm{d} \rho \\
\geq \sqrt{\frac{m_{1}}{m_{1}+1}} \int_{\sqrt{m_{1} \delta}}^{\infty} \eta(\rho ; x, \beta, \delta=0, d) \mathrm{d} \rho \\
\quad=\sqrt{\frac{m_{1}}{m_{1}+1}}\left(\int_{0}^{\infty}-\int_{0}^{\sqrt{m_{1} \delta}} \eta(\rho ; x, \beta, \delta=0, d) \mathrm{d} \rho\right) \\
\quad=\sqrt{\frac{m_{1}}{m_{1}+1}}\left(1-\frac{\int_{0}^{\sqrt{m_{1} \delta}} \eta(\rho ; x, \beta, \delta=0, d) \mathrm{d} \rho}{\int_{0}^{\infty} \eta(\rho ; x, \beta, \delta=0, d) \mathrm{d} \rho}\right) \\
\quad \geq \sqrt{\frac{m_{1}}{m_{1}+1}}\left(1-\frac{\sqrt{m_{1} \delta} \cdot \mathrm{I}_{0}\left(\frac{|d| \sqrt{m_{1} \delta}}{2 \sigma}\right)}{\sqrt{\frac{\pi \beta \sigma^{2}}{2\left(\beta+\sigma^{2}\right)}}} \eta(\rho ; x, \beta, \delta=0, d) \mathrm{d} \rho\right. \\
\quad
\end{aligned}
$$

where the first inequality follows from the non-negativity of the integrand; the subsequent inequality from (136); and the final inequality from (137) \& (138). The desired bound (83) now follows from (139) and (135).

\section{References}

[1] Richard E. Blahut, "Hypothesis testing and information theory," IEEE Trans. on Inform. Theory, vol. 20, no. 4, pp. 405-417, July 1974.

[2] I. Csiszár and J. Körner, Information Theory: Coding Theorems for Discrete Memoryless Systems, Academic Press, 1981.

[3] Suguru Arimoto, "Computation of random coding exponent functions," IEEE Trans. on Inform. Theory, vol. 22, no. 6, pp. 665-671, Nov. 1976.

[4] R. G. Gallager, Information Theory and Reliable Communication, John Wiley \& Sons, 1968. 
[5] G. Sh. Poltyrev, "Random coding bounds for discrete memoryless channels," Problems of Information Transmission, vol. 18, no. 1, pp. 12-26, January-March 1982.

[6] R. G. Gallager, "The random coding bound is tight for the average code," IEEE Trans. on Inform. Theory, pp. 244-246, March 1973.

[7] I. Csiszár, "The method of types," IEEE Trans. on Inform. Theory, vol. 44, no. 6, pp. 2505-2523, Oct. 1998.

[8] R. G. Gallager, "A simple derivation of the coding theorem and some applications," IEEE Trans. on Inform. Theory, vol. 11, pp. 3-18, January 1965.

[9] T. H. E. Ericson, "A Gaussian channel with slow fading," IEEE Trans. on Inform. Theory, vol. 16, no. 3, pp. 353-355, May 1970.

[10] W. Ahmed and P. McLane, "Random coding error exponents for twodimensional flat fading channels with complete channel state information," IEEE Trans. on Inform. Theory, vol. 45, pp. 1338-1346, May 1999.

[11] E. Biglieri, J. Proakis, and S. Shamai(Shitz), "Fading channels: information-theoretic and communications aspects," IEEE Trans. on Inform. Theory, vol. 44, no. 6, pp. 2619-2692, Oct. 1998.

[12] I. Abou-Faycal, M. Trott, and S. Shamai(Shitz), "The capacity of discrete time Rayleigh fading channels," IEEE Trans. on Inform. Theory, vol. 47, no. 4, pp. 1290-1301, May 2001.

[13] G. Taricco and M. Elia, "Capacity of fading channels with no side information," Electronics Letters, vol. 33, no. 16, pp. 1368-1370, July 31, 1997.

[14] A. Lapidoth and S. M. Moser, "Capacity bounds via duality with applications to multiple-antenna systems on flat fading channels," IEEE Trans. on Inform. Theory, vol. 49, no. 10, pp. 2426-2467, Oct. 2003.

[15] M. C. Gursoy, H. V. Poor, and S. Verdu, "The noncoherent Rician fading channel - part I: Structure of the capacity achieving input," IEEE Trans. on Wireless Communications, 2005.

[16] M. C. Gursoy, H. V. Poor, and S. Verdu, "The noncoherent Rician fading channel-part II: Spectral efficiency in the low power regime," IEEE Trans. on Wireless Communications, 2005. 
[17] B. C. Carlson and J.L. Gustafson, "Asymptotic expansions of the first elliptic integral," SIAM J. Math. Anal., pp. 1072-1092, 1985.

[18] I. S. Gradshteyn and I. M. Ryzhik, Table of Integrals, Series, and Products, Academic Press, San Diego, 5th edition, 1994.

[19] P. S. Byrd and M.D.Friedman, Handbook of Elliptic Integrals for Engineers and Scientists, Springer, second edition, 1971.

[20] George E. Andrews, Richard Askey, and Ranjan Roy, Special Functions, Encyclopedia of Mathematics and its Applications. Cambridge University Press, 1999. 